\title{
Molecular docking investigation of the amantadine binding to the enzymes upregulated or downregulated in Parkinson's disease
}

\author{
Mihaela lleana lonescu ${ }^{1,2}$ \\ ${ }^{1}$ Department of Microbiology, Iuliu Hațieganu University of Medicine and Pharmacy, 6 Louis Pasteur, 400349, Cluj- \\ Napoca, Romania, mionescu@umfcluj.ro \\ ${ }^{2}$ Department of Microbiology, County Emergency Clinical Hospital, 400006, Cluj-Napoca, Romania
}

E-mail: mionescu@umfcluj.ro;

Received: May 15, 2020; Revised: June 01, 2020; Published: June 15, 2020

\begin{abstract}
Parkinson's disease (PD) is a progressive neurodegenerative disease. Levodopa in combination with amantadine has a demonstrated efficacy in motility impairment. An extensive investigation of some enzymes described to be upregulated or downregulated in PD was made - adenylate kinase (AK), adenine phosphoribosyltransferase (APRT), ectonucleoside triphosphate diphosphohydrolase 1 (ENTPD1), nucleoside-diphosphate kinase 3 (NDK3), purine nucleoside phosphorylase 1 (PNP1), and ecto-5'nucleotidase (NT5E). Also, creatine kinase (CK) was included in the study because it is one of the main enzymes involved in the regulation of the nucleotide ratio in energy metabolism. To date, there is no proven link between amantadine treatment of PD and these enzymes. Because there are many AKs isoforms modified in PD, the AK was the first investigated. The molecular docking experiments allow the analysis of the selective binding of amantadine - unionized (with $-\mathrm{NH}_{2}$ group) and ionized form (with $\mathrm{NH}_{3}{ }^{+}$group) - to the AKs' isoforms implicated in PD. Using available X-ray 3D structures of human AKs in closed-conformation, it was demonstrated that there are notable differences between the interactions of the two forms of amantadine for the zebrafish AK1 (5XZ2), human AK2 (2C9Y), human AK5 (2BWJ), and AK from B.stearothermophilus. The cytosolic human AK1 and human AK2 mostly interact with ionized amantadine by AMP binding residues. The human AK5 interaction with ionized amantadine does not involve the residues from the catalytic site. Among other enzymes tested in the present study, APRT revealed the best results in respect of binding amantadine ionized form. The results offer a new perspective for further investigation of the connections between amantadine treatment of PD and some enzymes involved in purine metabolism.
\end{abstract}

C2020 by the authors. This article is an open-access article distributed under the terms and conditions of the Creative Commons Attribution license (http://creativecommons.org/licenses/by/4.0/).

\section{Keywords}

human adenylate kinases; adenine phosphoribosyltransferase; adenine metabolism; purine metabolism

\section{Introduction}

Parkinson's disease (PD) is an idiopathic neurodegenerative and progressive disease, which mainly produces motility impairment with bradykinesia, tremor, and postural instability [1,2]. In PD, the neurotransmitter dopamine is reduced as a result of the degeneration of the dopaminergic neurons from the substantia nigra [3]. The PD-related drug utilization and PD-related other degenerative diseases are 
due to the losses/impairment of the dopamine action at the basal ganglions [4,5]. Also, some toxins (6hydroxydopamine) are identified to induce dopaminergic cell death [6-8]. PD is a chronic disease with no curative treatment of the underline disease. Therefore is an ongoing interest in founding the most efficient strategy for treatment the motility disorders and other symptoms [9-14]. The drugs used in the treatment of PD should correct the dopamine/cholinergic balance, because the level of dopamine in the caudateputamen in the striatum is considerably reduced, with the exacerbation of the cholinergic control $[15,16]$. The most used dopaminergic drugs are levodopa, bromocriptine, and amantadine whereas the main anticholinergic drug is trihexyphenidyl [17-20].

Levodopa, discovered in 1950, was first approved in PD treatment by the FDA (U.S. Food and Drug Administration) in 1975 in combination with carbidopa, on the product called Sinemet [21]. An important side effect of long-term use of levodopa is levodopa-induced dyskinesia [22]. In this case, amantadine is the only treatment option that has been shown to be effective [23]. According to DrugBank (https://www.drugbank.ca/), amantadine (accession number DB00915) is not only an antiviral used for prophylaxis and treatment in infections with influenza $A$ but, also an effective antiparkinsonian in combination with levodopa. The mechanism of action of amantadine is not entirely understood. According to PubChem (https://pubchem.ncbi.nlm.nih.gov/), amantadine (PubChem CID 2130) stimulates the release of dopamine from striatal dopaminergic nerve terminals and inhibits the pre-synaptic uptake of dopamine.

In neurodegenerative diseases, among other biomarkers, some AKs isoforms are dysregulated. There is demonstrated that in stages 3-6 of the PD there is a down-regulation of the AK2, AK3 and AK4 in the substantia nigra. Also, there is a reduced expression of adenine phosphoribosyltransferase (APRT), ectonucleoside triphosphate diphosphorylase 1 (ENTPD1), nucleoside-diphosphate kinase 3 (NDK3) and purine nucleosise phosphorylase 1 (PNP1) mRNA. On contrary, the stages 5-6 of PD are characterized by the up-regulation of the AK1, AK5, nucleoside-diphosphate kinase 5 (NDK5), ecto-5'-nucleotidase (NT5E) and purine nucleoside phosphorylase 1(PNP1) [24]. As the changes in the purine metabolism influence the evolution of PD and, ultimately, the effectiveness of the treatment, the study of human AKs isoforms and enzymes involved in the purine metabolism is of great interest.

The present study aimed to analyze the connection between the dysregulated enzymes in different stages of PD and amantadine. It is noteworthy that different AKs isoforms are modified in PD. The elements that link the AK1 and AK2 isoforms and amantadine are dyskinesia and inflammation. AK1 and AK2 isoforms are altered in other muscle diseases - Duchenne muscular dystrophy - or in intense physical activity [25-30]. It has long been known, on the one hand, the positive role or amantadine in managing dyskinesia from PD and, on the other hand, the AK1 and AK2 dysregulation in PD. However, to date, there is no proven link between amantadine and AK in PD. Before planning a strategy that implies costly and time-consuming experiments, the molecular docking experiments were chosen to perform the present study.

The AK was the first enzyme analyzed because the specific conformational movements of the AKs during catalysis are well studied and there are many X-ray 3D structures of these conformations of the AKs deposed in the Protein Data Bank (PDB). Therefore, molecular docking, despite the limitations of the method, allows a first look at the connection between PD, amantadine, and some enzymes already demonstrated to be dysregulated in PD. We analyzed the amantadine ionized form (with $-\mathrm{NH}_{3}{ }^{+}$group) because the amantadine is ionized in physiological conditions [31].

To verify the hypothesis of that amantadine specifically target some enzymes modified in PD, the 
following enzymes were analyzed - adenylate kinase (AK), adenine phosphoribosyltransferase (APRT), ectonucleoside triphosphate diphosphohydrolase 1 (ENTPD1), nucleoside-diphosphate kinase 3 (NDK3), purine nucleoside phosphorylase 1 (PNP1), and ecto-5'-nucleotidase (NT5E). More, because in skeletal muscle AK1 and creatinine kinase (CK) are the main enzymes involved in the regulation of nucleotide ratio in energy metabolism, the molecular docking of CK was an additional analysis [27].

\section{Experimental}

The study workflow is as follows: (1) retrieving and analysis of the enzymes with X-ray 3D crystalline structure deposed in public databases, (2) molecular docking procedure with amantadine ionized form (with $-\mathrm{NH}_{3}{ }^{+}$group), (3) redocking of the substrates/substrates analogs with AKs, (4) multiple sequences alignment of AKs, (5) retrieving and analysis of the APRT, ENTPD1, NDK3, PNP1, NT5E, and CK with X-ray 3D crystalline structure deposed in public databases, (6) molecular docking procedure with amantadine ionized form (with $-\mathrm{NH}_{3}{ }^{+}$group) of the following enzymes: APRT, ENTPD1, NDK3, PNP1, NT5E, and CK.

\section{Adenylate kinase (AK)}

The human AKs isoforms and selected other AKs from other organisms were retrieved from the PDB (https://www.rcsb.org/). The characteristics of the AKs tested (PDB ID(s) 2C95, 1Z83, 5XZ2, 2C9Y, 2AR7, 2BBW, 2BWJ, 3HPQ, and 1ZIP) are synthesized in Table S1 [32-34].

\section{Human adenine phosphoribosyltransferase (APRT)}

The human APTRs co-crystallized with the substrate AMP and with adenine, allow a deep view of the amantadine interactions with the residues involved in catalysis The X-ray crystal structures of the APTRs included in the study (PDB ID(s) 1ORE, 1ZN7, 1ZN8, 1ZN9, 4X44, and 6FCI) are presented in the Table S2 [35-37].

\section{Human ecto-5'-nucleotidase (NT5E)}

The human NT5Es co-crystallized with the substrate adenosine and with phosphomethylphosphonic acid adenosyl ester, allow a deep view of the amantadine interactions with the residues involved in catalysis. The X-ray crystal structures of the NT5Es included in the study (PDB ID(s) 4H2I, 4H2G, 4H2F, and $4 \mathrm{H} 1 \mathrm{~S})$ are presented in the Table $\mathrm{S} 3[38,39]$.

\section{Human ectonucleoside triphosphate diphosphohydrolase 1 (ENTPD1)}

The human NDK3s in closed-conformation and without ligands allows a thorough analysis of the amantadine interactions with the catalytic site. The X-ray crystal structures of the NDK3s included in the study (PDB ID(s) 1ZS6, 2HVD, 2HVE, 1JXV, and 3BBB) are presented in the Table S4 [40-42].

\section{Human purine nucleoside phosphorylase 1 (PNP1)}

The many X-ray 3D structures of human PNP1 co-crystallized with the substrate or analag substrate allows a good interpretation of the molecular docking results of amantadine with PNP1. The X-ray crystal structures of the PNP1s included in the study (PDB ID(s) 1ULA, 1ULB, 2AOW, 2A0X, 2AOY, 1RSZ, and 1RFG) are presented in the Table S5 [43-45].

\section{Creatinin kinases (CK)}

Due to the different isoforms of the CKs, all these isoforms were selected. X-ray 3D structures of human CKs are compared to CKs of other organisms, due to the small number of human CKs structures deposed in 
PDB. The X-ray crystal structures of the CKs included in the study (PDB ID(s) 1CRK, 4Z9M, 2CRK, 1I0E, 1U6R, 3DRB, 3DRE, 1QH4, and 1GOW) are presented in the Table S6 [46-52].

\section{Inclusion and exclusion criteria}

Inclusion criteria: The human AKs and AKs form other eucaryotes with X-ray 3D structure in closedconformation - co-crystallized with substrate or analog substrate. For further comparison, AK1 from zebrafish (Danio rerio) and two bacterial AKs in closed-conformation were included - one from a Gramnegative bacteria, E.coli, and one from a Gram-positive bacteria, B.stearothermophilus. Other human enzymes described to be dysregulated in PD (APRT, NT5E, ENTPD1, NDK3, and PNP1). The human CKs were also tested because their major role in nucleotide ratios in muscles. When enzyme of human origin was not found in PDB, the same enzyme of other origin was retrieved.

Exclusion criteria: Human AKs in open conformation or with no X-ray 3D structure records in public databases. AKs from other organisms with the exception of the AKs selected in the inclusion criteria for further comparisons.

\section{Molecular docking procedure}

\section{Adenylate kinases preparation as receptors}

For the present study were selected from PDB Database (https://www.rcsb.org/) the AKs 3D structures in closed conformation - co-crystallized with substrate or analog substrate. The AKs X-ray 3D structures downloaded from the PDB Database as pdb files were prepared as receptors. All solvent molecules, the ions and the substrates were removed. In the case of multimeric AKs, only the chain that was crystallized with substrate or substrate analog was selected. Three chains were selected for human AK5 2BWJ - chains $B, C$, and $F$ - all three crystallized with AMP but with different interactions with AMP. Thus, in 3D crystal structure from PDB, two residues in the chain B form hydrophobic interaction with AMP (GIn29 and His39) and the Gln29 in the chain F forms a hydrophobic interaction with AMP and no interaction with AMP for chain C.

\section{Ligand preparation}

The 3D structure of ligands was retrieved from the PubChem Compound Database (https://www.ncbi.nlm.nih.gov/pccompound/). Molecular docking experiments were performed with the unionized amantadine (PubChem CID 2130) and ionized amantadine (with $-\mathrm{NH}_{3}{ }^{+}$) as ligand. The ionized amantadine was prepared in BIOVIA program by adding one hydrogen ion to the unionized amandatine structure. The redocking experiments were performed with the substrate/substrate analog with which the AKs were co-crystallized in the PDB; the 3D structure of the ligands was downloaded from the PDB Database as sdf file and visualize it on Dassault Systèmes BIOVA program - Discovery Studio Modeling Environment, Release 2017, San Diego: Dassault Systèmes, 2016 (http://accelrys.com). Further preparations for molecular docking were done by AutoDockTools.

\section{Molecular docking protocol}

Protein-ligand docking experiments were performed with the AutoDock4.2 program, distributed as open source under a GPL license (http://autodock.scripps.edu)[53-55]. The molecular docking data analysis was made on Windows platform using the Dassault Systèmes BIOVA program. Also, Cygwin DLL (cygwin1.dII) terminal was used for the running of molecular docking scripts on Windows platform. The molecular docking algorithm employed was with a rigid AKs structure and a ligand flexibility with 1-6 
number of torsion and aromaticity criterion at 7.5'. The searching parameter was the Lamarckian Genetic Algorithm (LGA), with a maximum of 2,500,000 energy evaluations. Other docking parameters were used as defaults.

\section{Multiple sequence alignment}

Multiple sequence alignment was performed by Clustal Omega program (https://www.ebi.ac.uk/$\underline{\text { Tools/msa/clustalo). }}$

\section{Results and Discussion}

The molecular docking of AKs with amantadine ionized form (with $-\mathrm{NH}_{3}{ }^{+}$group) and the redocking experiments with substrates or analog substrates

There are nine human AKs isoforms described until now, but only those isoforms with X-ray 3D structure solved were further analyzed by molecular docking. The results of human AKs were compared with AKs from other organisms. The AKs with 3D structure co-crystallized with substrates or analog substrates are optimal for our study because the AK is in closed-conformation, with the flexible LID domain covering the substrate. Because the study was designed based on the AKs in closed-conformation, the redocking experiments of AKs with its substrate or analog substrate were performed. The energy binding $(\Delta G)$ and the inhibition constant $\left(K_{\mathrm{i}}\right)$ of the best conformation of the complex AK-amantadine and the complex AK-ligand after redocking allowed a first view of the interaction of amantadine with AKs (Table 1).

Table 1. The energy binding $(\Delta G)$ and inhibition constant $\left(K_{\mathrm{i}}\right)$ of the best conformation of the complex AK amantadine and after redocking.

\begin{tabular}{|c|c|c|c|c|c|c|c|}
\hline \multirow{2}{*}{ AK } & \multirow[b]{2}{*}{ PDB ID } & \multicolumn{2}{|c|}{$\begin{array}{l}\text { Amantadine ionized } \\
\text { form }\end{array}$} & \multicolumn{2}{|c|}{$\begin{array}{l}\text { Amantadine unionized } \\
\text { form }\end{array}$} & \multicolumn{2}{|c|}{ Redocking $^{1}$} \\
\hline & & $\begin{array}{c}\Delta G \\
(\mathrm{kcal} / \mathrm{mol})\end{array}$ & $K_{\mathrm{i}}$ & $\begin{array}{c}\Delta G \\
(\mathrm{kcal} / \mathrm{mol})\end{array}$ & $K_{\mathrm{i}}$ & $\begin{array}{c}\Delta G \\
\text { (kcal/mol) }\end{array}$ & $K_{\mathrm{i}}$ \\
\hline \multicolumn{8}{|l|}{ Human cytosolic AKs } \\
\hline AK1 & $2 C 95$ & -6.19 & $29.04 \mu \mathrm{M}$ & -6.47 & $18.1 \mu \mathrm{M}$ & -10.95 & $9.36 \mathrm{nM}$ \\
\hline AK2 & $1 Z 83$ & -5.97 & $42.33 \mu \mathrm{M}$ & -6.05 & $36.94 \mu \mathrm{M}$ & -11.14 & $6.87 \mathrm{nM}$ \\
\hline AK5 & 2BWJ & -6.79 & $12.61 \mu \mathrm{M}$ & -7.03 & $7.03 \mu \mathrm{M}$ & -4.46 & $534.4 \mu \mathrm{M}$ \\
\hline \multicolumn{8}{|c|}{ Human mitochondrial AKs } \\
\hline AK2 & $2 \mathrm{C} 2 \mathrm{Y}$ & -6.00 & $39.68 \mu \mathrm{M}$ & -6.25 & $26.29 \mu \mathrm{M}$ & -7.38 & $3.91 \mu \mathrm{M}$ \\
\hline AK4 & 2BBW & \multicolumn{4}{|c|}{ Not performed } & Fail & \\
\hline \multicolumn{8}{|l|}{ Zebrafish AK } \\
\hline AK1 & $5 X Z 2$ & -5.51 & $91.53 \mu \mathrm{M}$ & -5.69 & $67.9 \mu \mathrm{M}$ & -13.2 & 209.44 pM \\
\hline \multicolumn{8}{|l|}{ Bacterial AKs } \\
\hline E.coli & $3 \mathrm{HOQ}$ & -6.55 & $15.78 \mu \mathrm{M}$ & -6.38 & $21.21 \mu \mathrm{M}$ & -5.62 & $75.52 \mu \mathrm{M}$ \\
\hline B.stearothermophilus & $1 \mathrm{ZIP}$ & -5.87 & $49.41 \mu \mathrm{M}$ & -5.77 & $59.42 \mu \mathrm{M}$ & -7.2 & $5.3 \mu \mathrm{M}$ \\
\hline
\end{tabular}

Redoking of the AKs isoforms with the substrates co-crystallized in the X-ray 3D structures

The redocking process allows verifying the docking parameters. Because the AK undergoes conformational changes during catalysis, the substrate does not perfectly fit with the PDB conformation on redocking experiments. One explanation is that the crystal structure was solved by an analog substrate, not by the AMP and ATP. The flexibility of the AK is very well studied. During the phosphoryl transfer, the two small peripheral domains of the AK (NMB binding domain and LID domain) move to close the ATP binding region. The AMP is tightly bound to the AMP site when the AMP domain undergoes minor movements. Contrary, when the ATP loosely bounds to the ATP site, the large movements of the LID domain occurs $\sim 30{ }^{\circ} \mathrm{C}$ and $88^{\circ}$ hinge bending rotation - then the LID domain closed tightly upon ATP [56]. However, 
observing the redocked conformations permits the chosen of the most suitable 3D structure when two closed-conformation of the same enzyme, co-crystallized with different substrates or analog substrates, was found in PDB. This is the case of AK isoform 1 for which there are three closed-conformation structures - two for human enzyme and one for zebrafish counterpart. The best redocked conformations are for the AKs co-crystallized with bis(adenosine)-5'-pentaphosphate (Ap5A) - 1X83 and 5XZ2 (Figure 1). The availability of three different conformation of the AK1 - an AK isoform dysregulated in PD - provides a substantial advantage for further analyses.

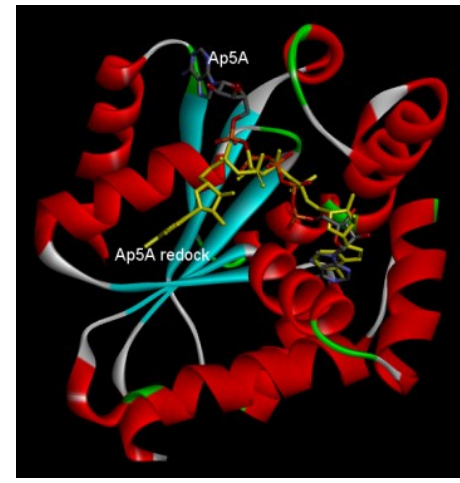

(a) 5 XZ2 (zebrafish AK1)

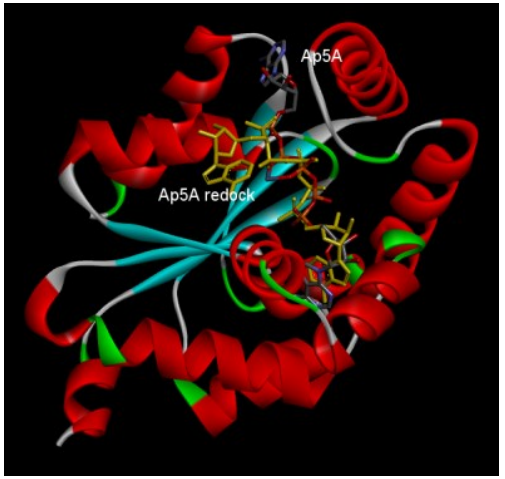

(b) $1 \times 83$ (human AK1)

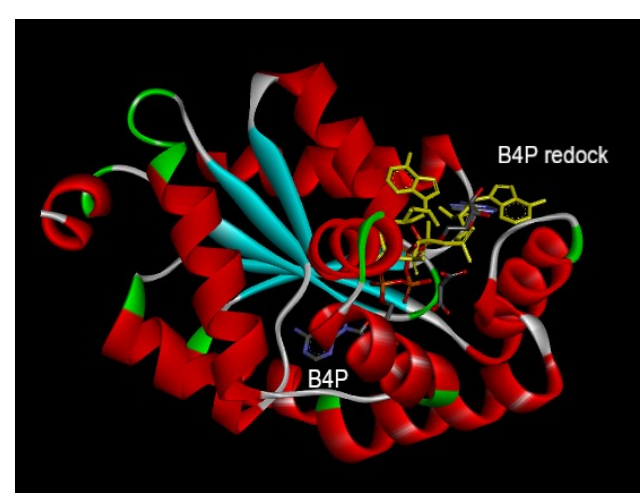

(c) 2 C95 (human AK1)

Figure 1. The comparative interactions of the AK1 isoforms with the analog substrates (Ap5A or B4P) after redocking. The redocked conformations are shown in yellow. (a) zebrafish AK1 (PDB ID 5XZ2); (b) human AK1 (PDB ID 1X83); (c) human AK1 (PDB ID 2C95).

The human AK2 isoform (PDB ID 2C9Y) is found in closed-conformation only co-crystallized with P1, P4$\mathrm{Di}$ (adenosine)tetraphosphate (B4P). The redocking of the B4P substrate as ligand demonstrate that the parameters specified in the docking method are reasonable even there is not a perfect match with the $X$ ray 3D structure from the PDB (Figure 2a). The human AK5 isoform (PDB ID 2BWJ) is deposed in PDB only co-crystallized with one substrate - AMP. Therefore, the enzyme is in semi closed-conformation. The redocking of the AMP does not show the same interactions as the X-ray 3D structure deposed in PDB (Figure 2b). The explanation could reside in the semi closed-conformation of the 2BWJ - the 3D structure contains only one substrate AMP, the substrate that causes minor movements of the AMP domain [56]. The semi-closed conformation of the human AK5 is one limitation of the present study, which relies on the X-ray 3D structure on closed-conformation.

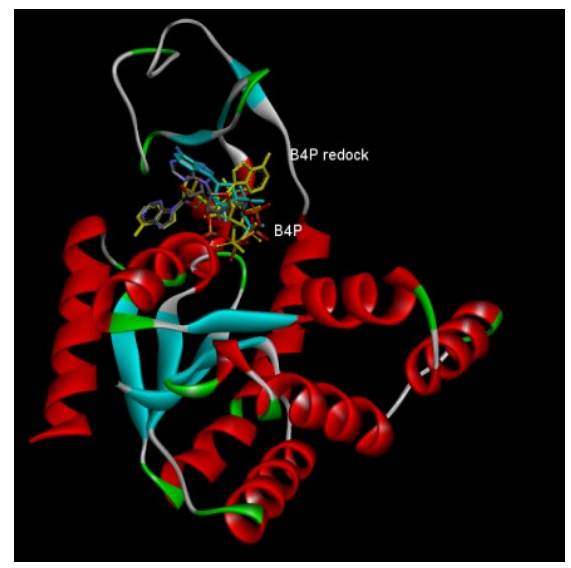

(a) $2 \mathrm{CgY}$ (human AK2)

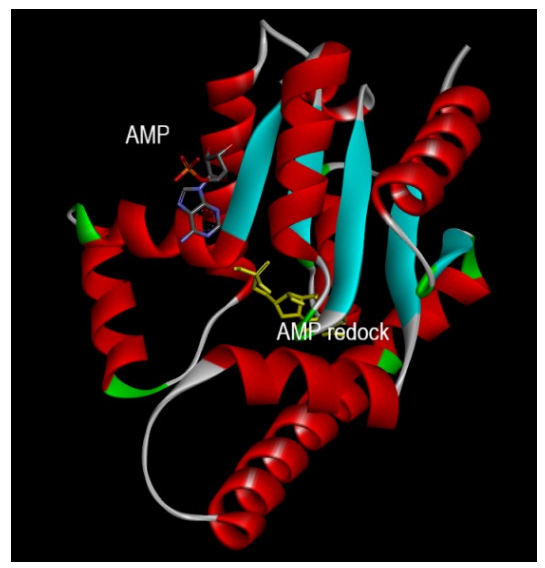

(b) 2BWJ (human AK5)

Figure 2. (a) The interactions comparative of the human AK2 (PDB ID 2C9Y) with the B4P after redocking; (b) The comparative interactions of the human AK5 (PDB ID 2BWJ) with the AMP after redocking. The redocked conformations are shown in yellow. 
The redocking of the analog substrate Ap5A as a ligand with AKs from E.coli and B.stearothermophilus does not perfectly match with the conformation of the Ap5A from the X-ray 3D structure deposed in PDB (Figure 3). Despite this limitation, the closed-conformation of $A K$ is the best choice when performing molecular studies involving this enzyme. Furthermore, the present study's results allow the comparison of the interaction of the human AKs and bacterial AKs with amantadine.

The AK4 is also named AK3-like and is a GTP:ATP phosphotransferase (EC 2.7.4.10) [57-59]. The mitochondrial human AK4 (PDB ID 2BBW) was deposed on PDB in closed-conformation co-crystallized with diguanosine-pentaphosphate (GP5). The AK4 has some characteristics that differentiate it from other AK isoforms, the most significant being its catalytic activity (a ribonucleoside 5'-diphosphate $+A T P=a$ ribonucleoside 5'-triphosphate + ADP) (https://www.uniprot.org/uniprot/P27144). Because the parameters in the input file do not allow substrate redocking, the molecular docking of amantadine with AK4 was not made.

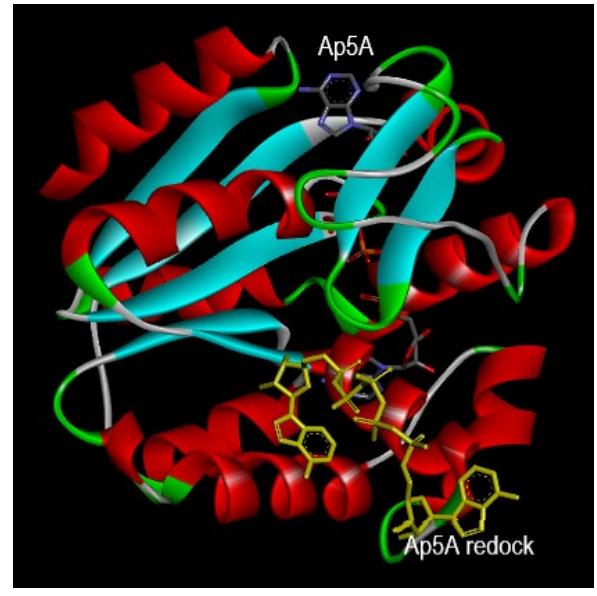

(a) $3 \mathrm{HPQ}$ (E.coli AK)

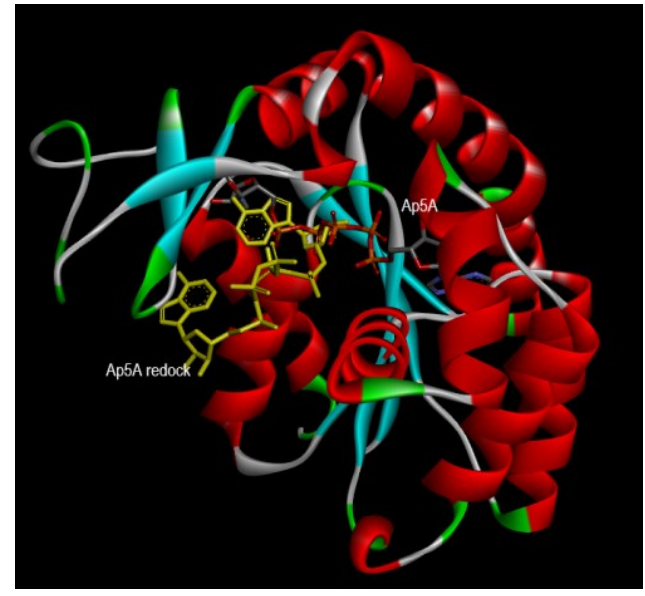

(b) 1ZIP (B.strearothermophilus AK)

Figure 3. The comparative interactions of the bacterial AKs with the analog substrate Ap5A after redocking. The redocked conformations are shown in yellow. (a) E.coli AK (PDB ID 3HPQ); (b) B.stearothermophilus AK (PDB ID 1ZIP).

\section{Molecular docking of ionized amantadine with AK1}

The three X-ray 3D structures of the AK1 were analyzed for their interaction with ionized amantadine (with $-\mathrm{NH}_{3}{ }^{+}$group). The human AK1 co-crystallized with Ap5A (PDB ID 1Z83) mostly interacts with ionized amantadine with the residues involved in AMP binding. Thus, the enzyme forms van der Waals forces with Thr39, Tyr95, and Pro96; conventional hydrogen bonds with Gly94 and GIn101; and an unfavorable positive-positive interaction with Arg97. In total, there are five residues from the NMP binding region that interact with ionized amantadine - Thr39, Gly40, Leu43, Leu66, and Val67 (Figure 4). A comparison of the AK1 (PDB ID 1Z83) interactions with the two forms of amantadine, reveals that the group $-\mathrm{NH}_{3}{ }^{+}$of the ionized amantadine is involved in three conventional hydrogen bonds like the $-\mathrm{NH}_{2}$ group of the unionized form. The difference lies in the residues involved in these interactions - Val67 interacts with $-\mathrm{NH}_{2}$ of unionized amantadine, respectively $\mathrm{G} \ln 94$ and $\mathrm{G} \ln 101$ with $-\mathrm{NH}_{3}{ }^{+}$of ionized amantadine. 


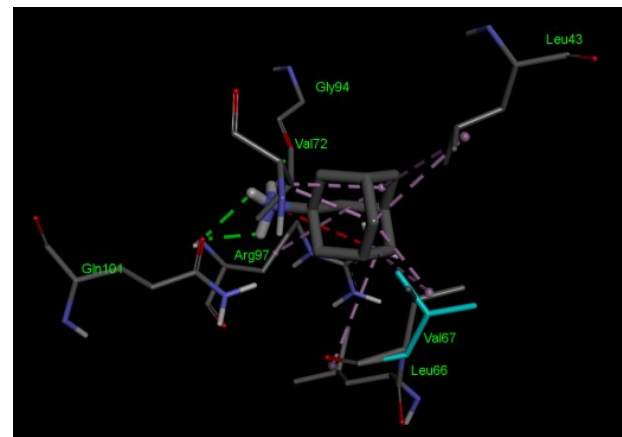

(a)

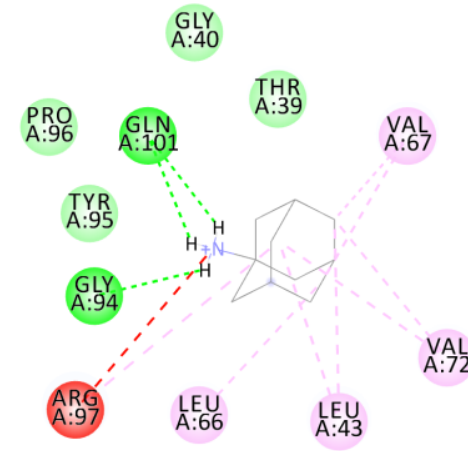

(b)

\begin{tabular}{cc}
\hline Gln101:OE1-H27 & $2.26 \AA$ \\
\hline Gln101:OE1-H5 & $2.09 \AA$ \\
Gly94:O-H27 & $1.92 \AA$ \\
Leu43 & $4.93 \AA$ \\
Leu66 & $4.48 \AA$ \\
Val67 & $4.68 \AA$ \\
Val72 & $5.05 \AA$ \\
Arg97 & $5.46 \AA$ \\
Leu43-C9 & $4.34 \AA$ \\
Val67-C9 & $3.78 \AA$ \\
Val72-C9 & $3.74 \AA$ \\
\hline Arg97:NH2-N1 & $5.46 \AA$
\end{tabular}

(c)

Figure 4. Interactions of ionized amantadine with human AK1, PDB ID 1Z83. (a) 3D display of ionized amantadine interaction as ligand with the $1 Z 83$ residues; (b) code color for interactions: in green are shown conventional hydrogen bonds, light green - van der Waals forces, mauve - alkyl hydrophobic interactions, red - unfavorable positive-positive interactions; (c) the distances $(\AA)$ of the $1 Z 83$ - ionized amantadine interactions.

The human AK1 co-crystallized with B4P (PDB ID 2C95) forms an electrostatic interaction between Glu62, a residue belonging to NMP binding region, and $-\mathrm{NH}_{3}{ }^{+}$group of ionized amantadine. The same residue, Glu62, interacts with the $-\mathrm{NH}_{2}$ group of the unionized amantadine by two conventional hydrogen bonds. In contrast with human AK1 (PDB ID 1Z83), the human AK1 (PDB ID 2C95) interacts with ionized amantadine with residues belonging to NMP binding region (Arg44, Val47, Ser58, Met61, and Glu62) and LID domain (Gly137, Arg138, Val139, and Asp140). More, AK1 (PDB ID 2C95) interacts with only two AMP binding residues - Arg44 and Arg138 (Figure 5).

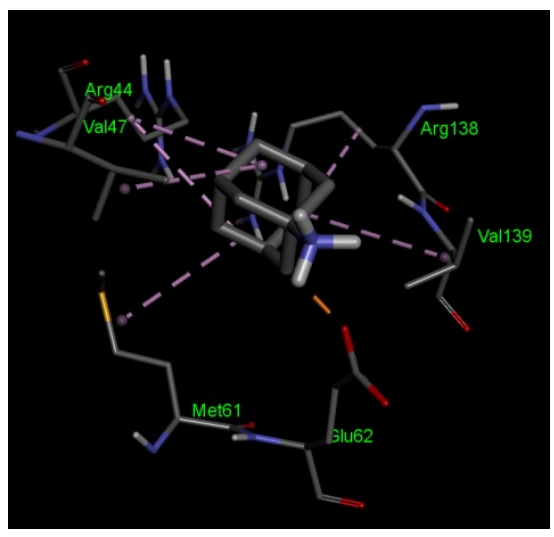

(a)

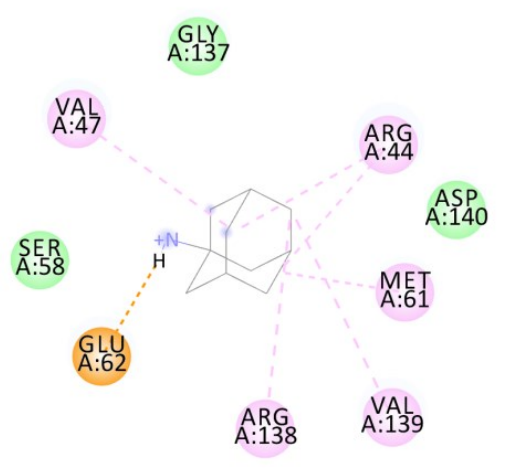

(b)

$\begin{array}{cc}\text { Glu62:OE2-H27 } & 1.75 \AA \\ \text { Arg44 } & 4.10 \AA \\ \text { Arg44 } & 4.63 \AA \\ \text { Val47 } & 5.33 \AA \\ \text { Met61 } & 4.12 \AA \\ \text { Arg138-C9 } & 4.45 \AA \\ \text { Val139-C9 } & 4.55 \AA\end{array}$

(c)

Figure 5. Interactions of ionized amantadine with human AK1, PDB ID 2C95. (a) 3D display of ionized amantadine interaction as ligand with the 2C95 residues; (b) code color for interactions: in orange are shown the salt bridge, light green - van der Waals forces, mauve - alkyl hydrophobic interactions; (c) the distances

( $)$ of the 2C95 - ionized amantadine interactions.

The zebrafish AK1 co-crystallized with Ap5A (PDB ID 5XZ2) shows much more differences regarding the interactions with ionized amantadine, no residue which interacts with unionized amantadine interact with ionized amantadine. In contrast with unionized amantadine, the $-\mathrm{NH}_{3}{ }^{+}$group of the ionized amantadine interacts with the residues involved in catalysis - one residue from the NMP binding region, Met61, forms a conventional hydrogen bond and Asp140 from the LID domain forms two electrostatic interactions. Similar to human AK1 (PDB ID 2C95), the zebrafish AK1 interacts with ionized amantadine by residues from the NMP binding region (Leu43, Met61, Gly64, Glu65, Leu66, and Val67) and with residues from the LID 
region (Arg138 and Asp140). Most of the residues that interact with ionized amantadine are AMP binding residues - Gly64, Glu65, Leu66, Val67, Arg 138, and Arg 149 (Figure 6).

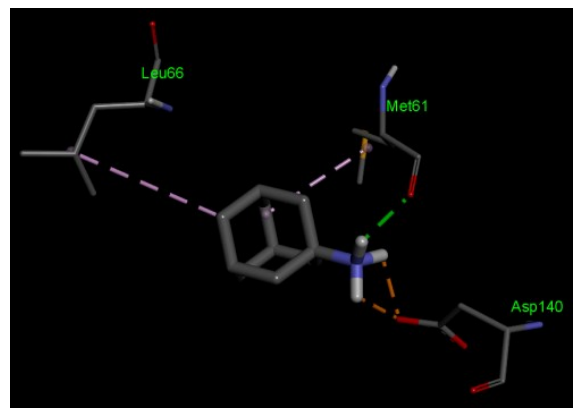

(a)

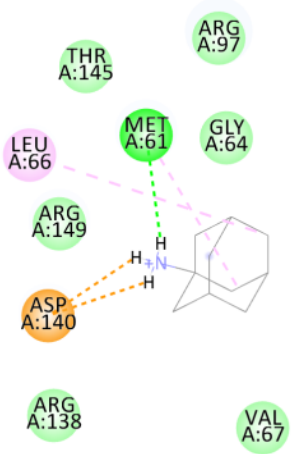

(b)

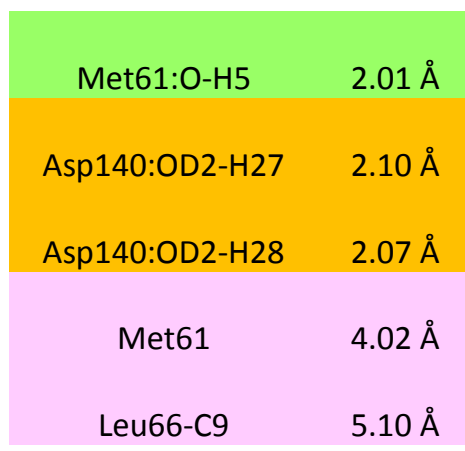

(c)

Figure 6. Interactions of ionized amantadine with zebrafish AK1, PDB ID 5XZ2. (a) 3D display of ionized amantadine interaction as ligand with the $5 X Z 2$ residues; (b) code color for interactions: in green are shown conventional hydrogen bonds, orange - salt bridge, light green - van der Waals forces, mauve - alkyl hydrophobic interactions; (c) the distances $(\AA ̊)$ of the 5XZ2 - ionized amantadine interactions.

\section{Molecular docking of ionized amantadine with human AK2}

Human AK2 (PDB ID 2C9Y) shows different interactions with the two forms of amantadine. $\mathrm{The}^{-} \mathrm{NH}_{3}{ }^{+}$ group forms a conventional hydrogen bond with a residue from the AMP binding region (Val74) and two electrostatic interactions with Asp76. Two residues from the AMP binding region form alkyl interactions (Arg103 and Phe101). Unlike unionized amantadine, the ionized amantadine shows a better match with the residues from the catalytic site. Ionized amantadine interacts with five AMP binding residues - Thr44, Val74, Phe101, Arg103, and Gln107 (Figure 7).

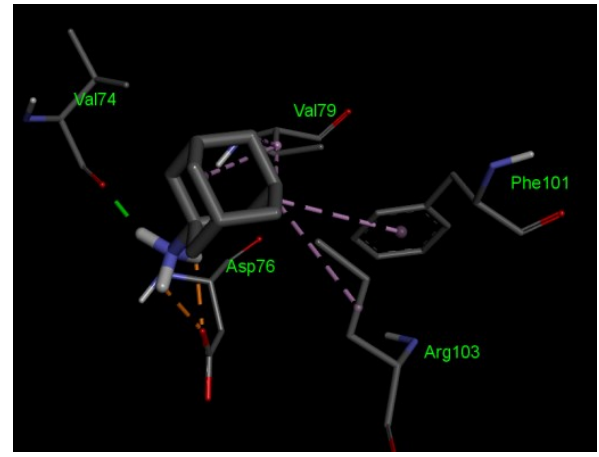

(a)

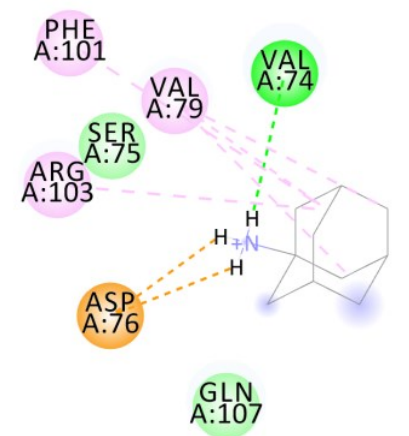

(b)

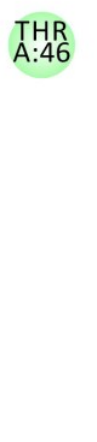

\begin{tabular}{cc} 
Val74:O-H5 & $1.96 \AA$ \\
\hline Asp76:OD1-H27 & $2.17 \AA$ \\
Asp76:OD1-H28 & $2.15 \AA$ \\
Val79 & $5.40 \AA$ \\
Val79 & $4.46 \AA$ \\
Val79-C9 & $3.69 \AA$ \\
Arg103 & $4.76 \AA$ \\
Phe101 & $4.64 \AA$
\end{tabular}

(c)

Figure 7. Interactions of ionized amantadine with human AK2, PDB ID 2C9Y. (a) 3D display of ionized amantadine interaction as ligand with the 2C9Y residues; (b) code color for interactions: in green are shown conventional hydrogen bonds, orange - salt bridge, light green - van der Waals forces, mauve - alkyl hydrophobic interactions; (c) the distances $(\AA)$ of the 2C9Y-ionized amantadine interactions.

\section{Molecular docking of ionized amantadine with human AK5}

Unlike unionized amantadine, the ionized amantadine interacts with human AK5 (PDB ID 2BWJ-chain B) residues that do not belong to the domains involved in catalysis. The van der Waals forces are observed with two glycines in the phosphate-binding loop (P-loop) (Figure 8). 


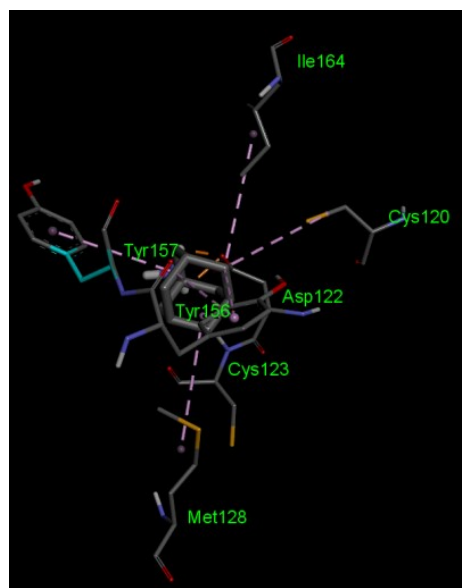

(a)

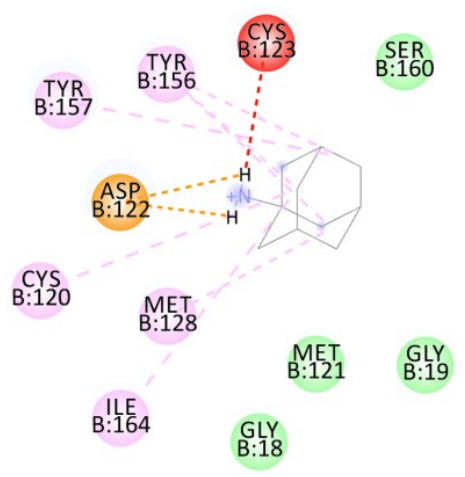

(b)

\begin{tabular}{cc} 
Asp122:OD1-H27 & $1.98 \AA$ \\
Asp122:OD1-H5 & $2.17 \AA$ \\
\hline Met128 & $5.35 \AA$ \\
Cys120-C10 & $5.26 \AA$ \\
Ile164-C10 & $5.40 \AA$ \\
Tyr156 & $4.65 \AA$ \\
Tyr156 & $4.66 \AA$ \\
Tyr156-C10 & $4.40 \AA$ \\
Tyr157 & $5.08 \AA$ \\
\hline Cys123:HN-H5 & $2.35 \AA$ \\
\hline
\end{tabular}

(c)

Figure 8. Interactions of amantadine with human AK5, PDB ID 2BWJ. (a) 3D display of ionized amantadine interaction as ligand with the 2BWJ residues; (b) code color for interactions: in orange are shown salt bridge, light green - van der Waals forces, mauve - alkyl hydrophobic interactions, red - unfavorable donor-donor interactions; (c) the distances (Å) of the 2BWJ - ionized amantadine interactions.

\section{Molecular docking of ionized amantadine with bacterial AKs}

A comparison of human AKs with their bacterial counterparts was necessary to draw a valid conclusion about the specificity of human AKs for amantadine. Bacterial AKs with X-ray 3D structure deposited in PDB are much numerous than AKs from eucaryotes. AK from E.coli (PDB ID 3HPQ) interacts with ionized amantadine through residues that do not belong to the catalytic site. Only Ala8 (from the P-loop) forms two alkyl hydrophobic interactions with ionized amantadine (Figure 9).

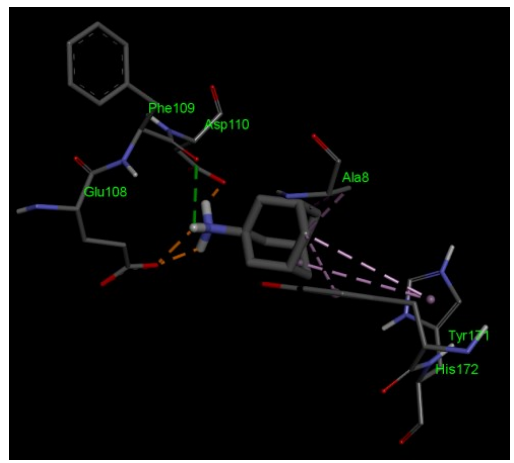

(a)

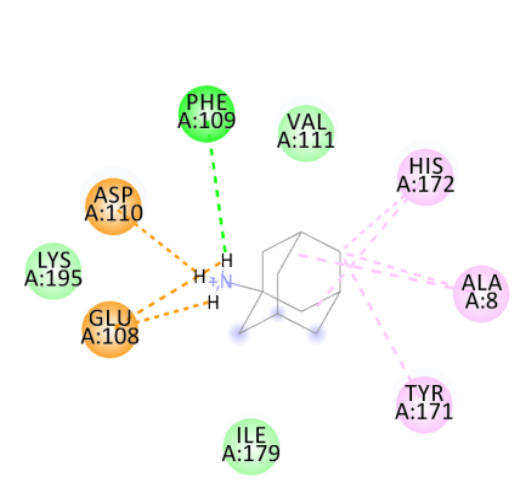

(b)

\begin{tabular}{cc} 
Phe109:O-H5 & $3.07 \AA$ \\
\hline Glu108:OE2-H27 & $2.16 \AA$ \\
Glu108:OE2-H25 & $2.13 \AA$ \\
Asp110:OD1-H28 & $1.89 \AA$ \\
Ala8 & $4.31 \AA$ \\
Ala8-C9 & $3.27 \AA$ \\
Tyr171-C9 & $4.30 \AA$ \\
His172 & $5.36 \AA$ \\
His172-C9 & $5.39 \AA$
\end{tabular}

(c)

Figure 9. Interactions of ionized amantadine with AK from E.coli, PDB ID 3HPQ. (a) 3D display of amantadine interaction as ligand with the $3 \mathrm{HPQ}$ residues; (b) code color for interactions: in green are shown conventional hydrogen bonds, orange - salt bridge, light green - van der Waals forces, mauve - alkyl hydrophobic interactions; (c) the distances $(\AA)$ of the $3 \mathrm{HPQ}$ - ionized amantadine interactions.

AK from B. stearothermophilus (PDB ID ZIP) interacts with ionized amantadine from two residues belonging to the LID region - with Arg162 by two electrostatic interactions and with Arg160 by van der Waals forces. The Pro9 from the P-loop forms alkyl interactions with ionized amantadine. Also, four residues from the NMP binding region interact with ionized amantadine - Met53, Leu58, Gly56, and Arg171. More, five AMP-binding residues interact with ionized amantadine - Gly56, Asp57, Leu58, Arg88, and Arg171 (Figure 10). 


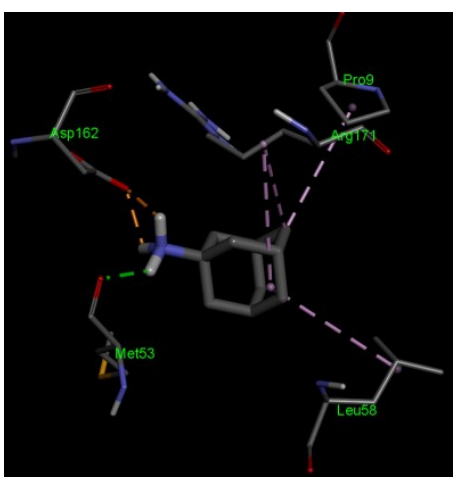

(a)

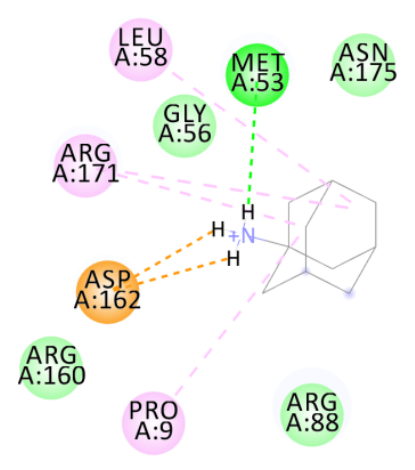

(b)

\begin{tabular}{cc} 
Met53:O-H5 & $2.07 \AA$ \\
\hline Asp162:OD2-H27 & $2.12 \AA$ \\
Asp162:OD2-H28 & $2.15 \AA$ \\
\hline Arg171 & $5.23 \AA$ \\
Leu58 & $4.94 \AA$ \\
Pro9-C10 & $4.78 \AA$ \\
Arg171-C10 & $4.41 \AA$
\end{tabular}

(c)

Figure 10. Interactions of ionized amantadine with AK from B.stearothermophilus, PDB ID 1ZIP. (a) 3D display of amantadine interaction as ligand with the 1ZIP residues; (b) code color for interactions: in green are shown conventional hydrogen bonds, orange - salt bridge, light green - van der Waals forces, mauve - alkyl hydrophobic interactions; (c) the distances $(\AA)$ of the 1ZIP - ionized amantadine interactions.

\section{Comparison of molecular docking of ionized amantadine with the AKs studied}

The overall comparison of the molecular docking results offers a better view of the interactions of the two forms of amantadine with the human and bacterial AKs. As the physiological form of amantadine is ionized, the comparison of molecular docking results with both forms of the drug has advanced the study on the hypothesis of the AK - amantadine - PD connection. Among the AKs included in the present study the zebrafish AK1 (PDB ID 5XZ2), human AK2 (PDB ID 2C9Y), human AK5 (PDB ID 2BWJ), and AK from B.stearothermophilus (PDB ID 1ZIP) show the most differences in interactions with the two forms of amantadine (Figure 11).

\section{Multiple sequence alignment of the AKs isoforms}

The multiple sequence alignment allows an overview of the interactions of the two forms of amantadine with the AKs isoforms. The residues involved substrate binding are well conserved among bacterial AKs and eucaryotes AKs. The interaction of unionized amantadine with zebrafish AK1 and human AK4 mainly involves the residues from the LID region that interact with unionized amantadine. In contrast, the human AK1, and AK5 interact with unionized amantadine through AMP binding residues. Minor similarities are noticed between human AK2 and AK from E.coli, both of which have two residues in the phosphate-binding loop ( $\mathrm{P}$-loop) that interact with unionized amantadine - one residue glycine being identical for the two enzymes. Also, human AK1 shows some similarities with human AK4 with three different residues from the LID region.

An in-depth analysis of the two forms of ionized amantadine shows that, except for AK5 (PDB ID 2BWJ), the ionized amantadine interacts predominantly with AMP binding residues. Among AK1 isoforms, the interactions with ionized amantadine are not similar. The AK2 (PDB ID 2C9Y) and the B.stearothermophilus AK (PDB ID 1ZIP) show a better match with the residues at the catalytic site with the ionized amantadine. Unlike unionized amantadine, the ionized amantadine interacts with more AMP binding residues of AK1 and AK2 isoforms (Figure 12). 


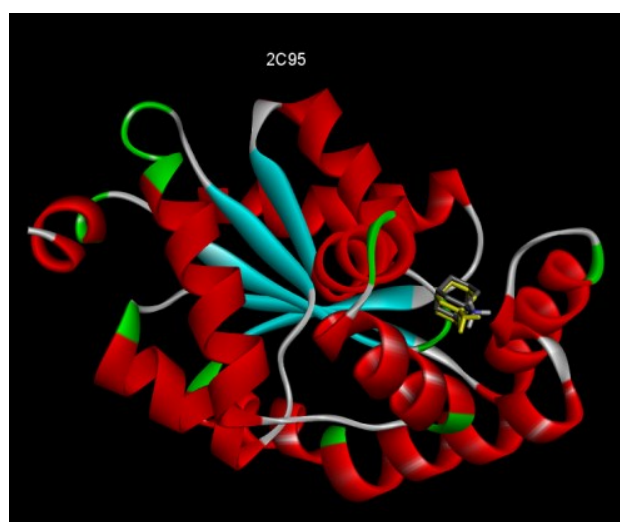

(a)

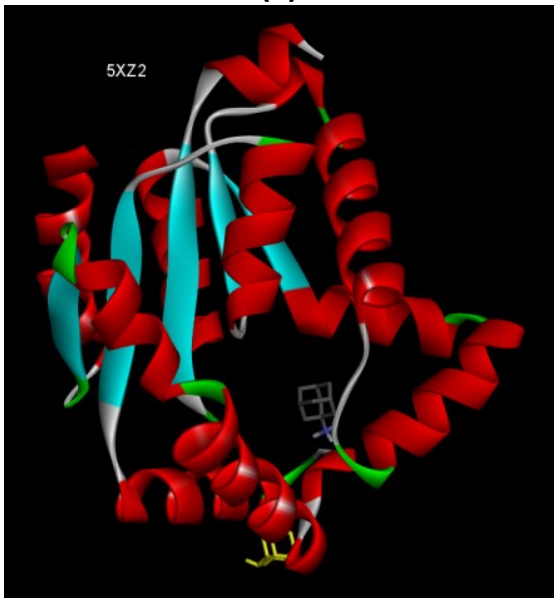

(c)

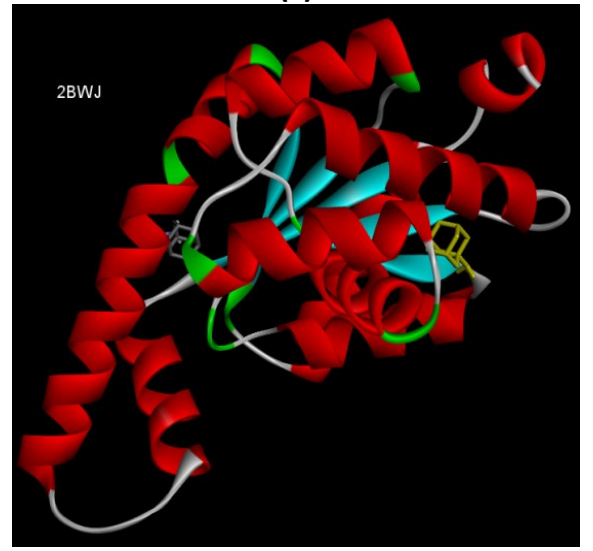

(e)

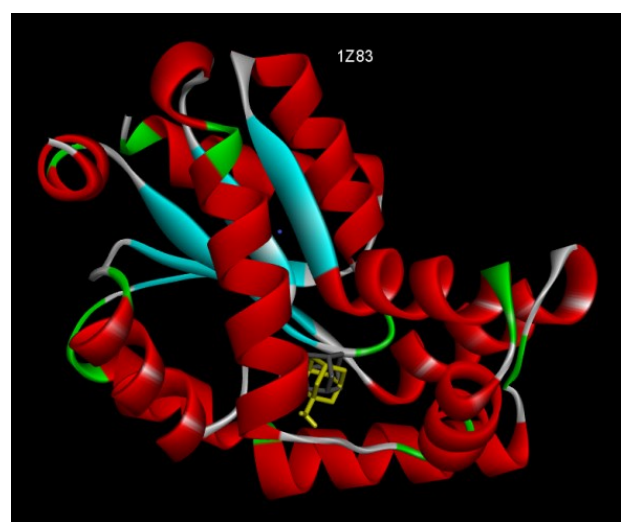

(b)

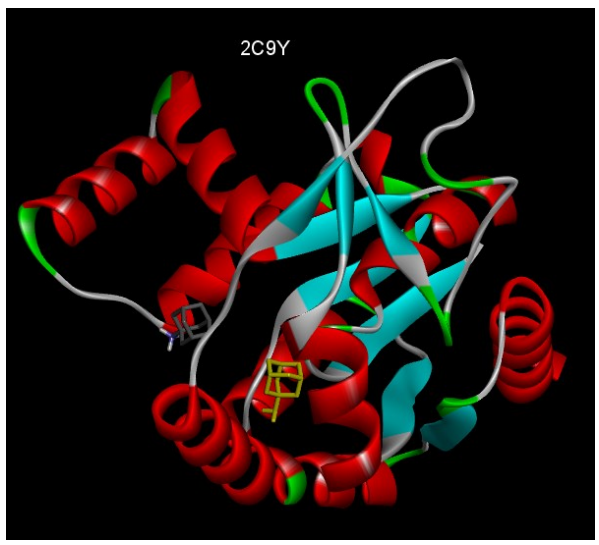

(d)

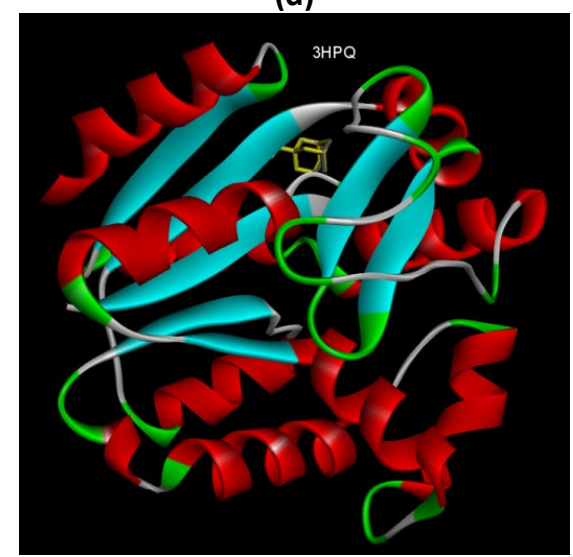

(f)

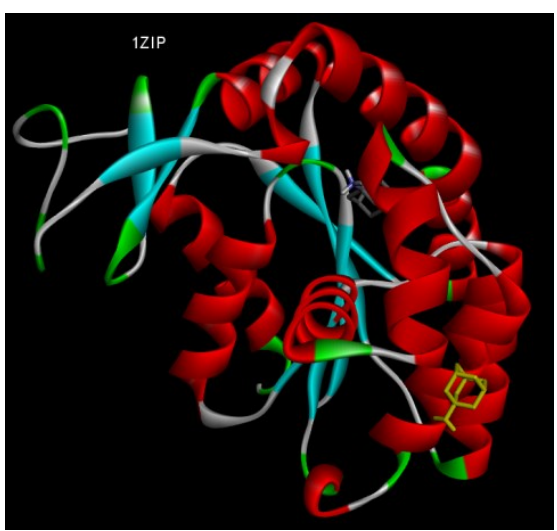

(g)

Figure 11. Interactions of unionized and ionized amantadine with AKs. (a) human AK1 (PDB ID 2C95); (b) human AK1 (PDB ID 1Z85) (c) zebrafish AK1 (PDB ID 5XZ2); (d) human AK2 (PDB ID 2C9Y); (e) human AK5 (PDB ID 2BWJ) (f) AK from E.coli (PDB ID 3HPQ); (g) AK from B.stearothermophilus (PDB ID 1ZIP); unionized amantadine is shown in yellow. 


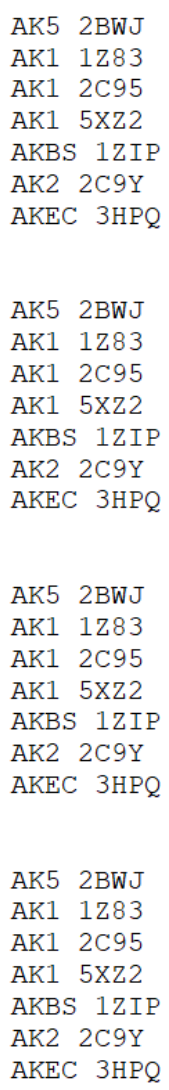

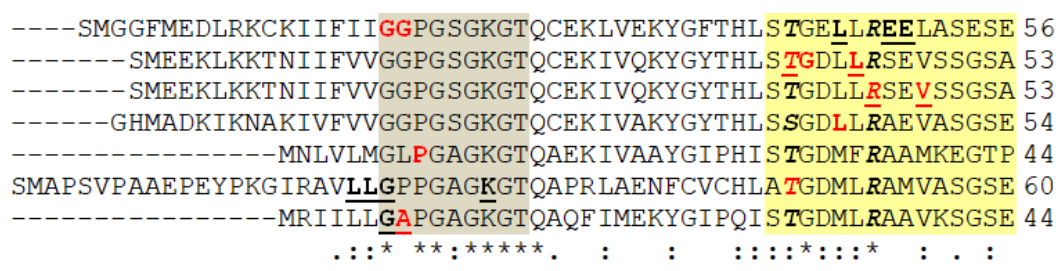

RSKLIRD IMERGDLVP SGIVLELLKEAMVASLGDTRGFLIDGYPREVKQGEEFGRRIGD- 115 RGKKLSE IMEKGQLVPLETVLDMLRDAMVAKVNTSKGFLIDGYPREVQQGEEFERRIGQ- 112 RGKKLSE IMEKG QLVP LETVLDMLRDAMVAKVNTS KGFLID GYPR EVQ QGEEFERRIGQ- 112

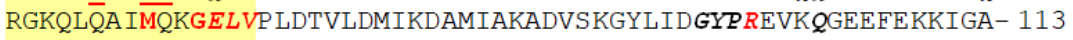
LGLQAKQYMDRG DLVP DEVTIGIVRERL-SKDDCQNGFLLD GFPRTVAQAEALE TMLADI 103 LGKKLKATMDAGKLVSDEMVVELIEKNL-ETPLCKNGFLLDGFPRTVRQAEMLDDLMEKR 119 LGKQAKD IMDAGKLVT DELVIALVKERI-AQEDCRNGFLLD GFPRT I PQADAM----KEA 99 ${ }^{\star}:{ }^{\star} \cdot{ }^{\star \star} \quad .::: . .: \quad . \quad{ }^{\star}:{ }^{\star}:{ }^{\star \star}:{ }^{\star \star}:{ }^{\star} .:$ :

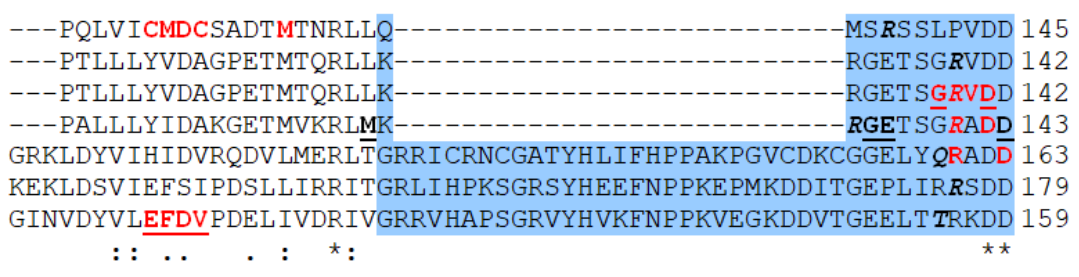

TTKT IAKRLEAYYRAS IPVIAYYETKTQLHKINAEGTPED-VFLQLCTAIDSIFL----- 199 NEETIKKRLETYYKATEPVIAFYEKRGIVRKVNAEGSVDS-VFSQVCTHLDALLN----- 196 NEET IKK RLETYYKATEPVIAFYEKRGIVRKVNAEGSVDS-VFSOVCTHLDALLN----- 196 NEET IKK RLDLYYKATEPVIAFYEQRGIVRKINSELPVDE-VFAIVEKAIDELK------ 196 NEATVAN RLEVNMKQMKPLVDFYEQKGYLRNINGEQDM-EKVFAD IRELLGGLAR----- 217 NEKALKI RLQAYHTQT TPLIEYYRKRGIHSAIDASQTPDV-VFAS I LAAFSKATCKDLVM 238 QEETVRK RLVEYHQMTAPLIGYYSKEAEAGNTKYAKVDGTKPVAEVRADLEKI------- 212

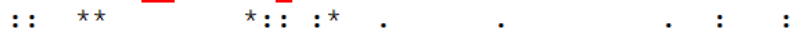

Figure 12. Multiple sequence alignment of the AKs sequences. In gray background are marked the residues belong to Walker A motif - phosphate-binding loop or P-loop; in yellow background is marked the NMP binding region and the residues that belong to AMP binding region are italic-bolded; in blue background is marked the LID region; with red are marked the residues (van der Waals, conventional hydrogen bond or alkyl interactions) which interact with amantadine ionized form (with $-\mathrm{NH}_{3}{ }^{+}$group); the residues which interact with amantadine unionized form (with $-\mathrm{NH}_{2}$ group) are bolded-underlined; the residues which interact with amantadine unionized and ionized are red and bolded-underlined; AKEC is AK from E.coli; AKBS is AK from B.stearothermophilus; with dot "." are marked the semi-conservative replacements; with colon ":" are marked the conservative replacements; with "*" are marked the identities of the residues.

Molecular docking of the human adenine phosphoribosyltransferase with ionized amantadine and the redocking with the substrates

The human APRTs (E.C. 2.4.2.7) found in PDB were analyzed for the amantadine interactions. The enzyme catalyses the reaction: AMP + diphosphate $=5$-phospho-alpha-D-ribose 1-diphosphate + adenine. In the adenine metabolism, APRT is involved in the first stage of the subpathway that synthesizes AMP from adenine (UniProtKB P07741). Thus, in adenine metabolism, APRT is closely connected with AK by the substrate AMP. Therefore, the APRT was the first enzyme tested to verify the hypothesis that amantadine specifically binds some human AKs isoforms. First, the redock of the substrates co-crystallized in the X-ray 3D structures was made. Then, amantadine ionized form was tested and the interactions with the residues from the catalytic site (Table2).

Among the four PDB structure co-crystallized with adenosine monophosphate (AMP) (PDB ID(s) 1ORE, $1 Z N 8,1 Z N 9$, and 4X44), two of them show the best interaction of amantadine with the AMP-binding residues. The amantadine -1 ZN9 interactions involve four residues that also bind AMP (Figure 13). 
Table 2. The energy binding $(\Delta G)$ and inhibition constant $\left(K_{\mathrm{i}}\right)$ of the best conformation of the complex APRT - amantadine (ionized form) and after redocking.

\begin{tabular}{ccccc}
\hline & \multicolumn{2}{c}{ Amantadine } & \multicolumn{2}{c}{ redocking $^{1}$} \\
PDB ID & $\begin{array}{c}\Delta \boldsymbol{G} \\
(\mathbf{k c a l} / \mathrm{mol})\end{array}$ & $\boldsymbol{K}_{\mathrm{i}}(\boldsymbol{\mu M})$ & $\begin{array}{c}\Delta \boldsymbol{G} \\
(\mathbf{k c a l} / \mathrm{mol})\end{array}$ & $\boldsymbol{K}_{\mathrm{i}}(\boldsymbol{\mu M})$ \\
\hline 1ORE & -6.44 & 18.93 & -6.91 & 8.68 \\
1ZN9 & -6.52 & 16.77 & -6.3 & 24.04 \\
4X44 & -6.01 & 39.53 & -8.1 & 1.15 \\
1ZN8 & -6.15 & 31.21 & -7.39 & 3.83 \\
6FCl & -7.33 & 4.26 & -6.21 & 28.14 \\
1ZN7 & -6.9 & 8.73 & -5.58 & 81.74 \\
\hline
\end{tabular}

${ }^{1}$ redocking with the substrates co-crystallized in PDB X-ray 3D structures.

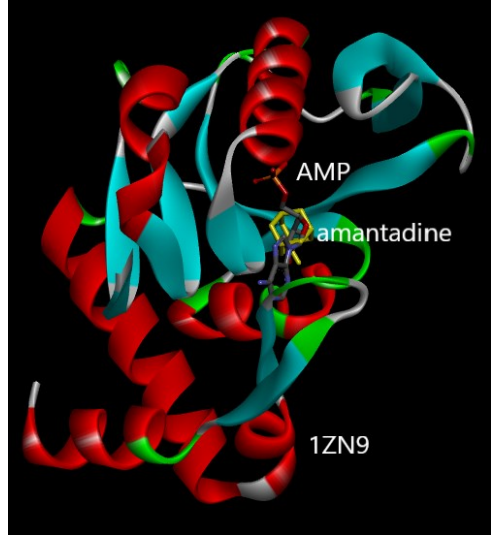

(a)

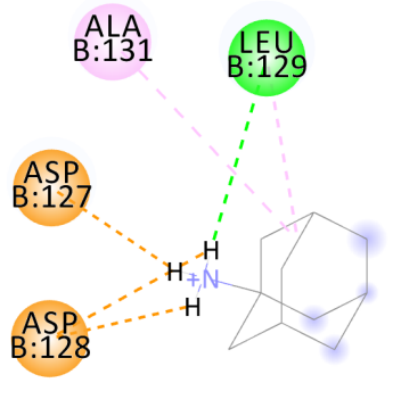

(b)

\begin{tabular}{cc}
\hline Leu129:O-H5 & $3.06 \AA$ \\
\hline Asp127:OD2-H28 & $1.84 \AA$ \\
Asp128:OD1-H27 & $2.04 \AA$ \\
Asp128:OD1-H5 & $2.14 \AA$ \\
\hline Ala131 & $4.03 \AA$ \\
Leu129 & $4.08 \AA$
\end{tabular}

(c)

Figure 13. Interactions of ionized amantadine with APRT co-crystallized with AMP, PDB ID 1ZN9. (a) 3D display of the amantadine (shows in yellow) and AMP in binding pocket; (b) code color for interactions: in green are shown conventional hydrogen bonds, orange - salt bridge, mauve - alkyl hydrophobic interactions; (c) the distances $(\AA)$ of the 1ZN9 - ionized amantadine interactions.

Similarly, amantadine - 1ZN8 interactions involve seven AMP-binding residues. Among them, four residues are the same observed in amantadine - 1ZN9 interactions (Asp127, Asp128, Leu129, and Ala131)

(Figure 14).

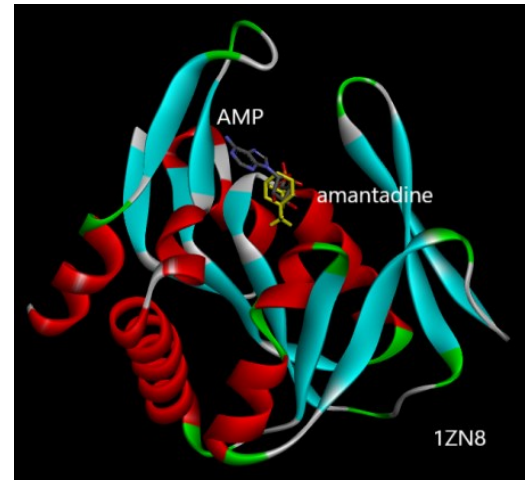

(a)

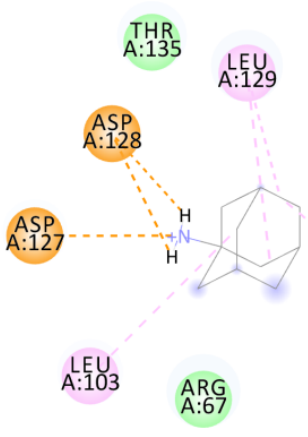

(b)

\begin{tabular}{|ccc} 
& Asp127:OD2-N1 & $2.68 \AA$ \\
& Asp128:OD2-H27 & $2.23 \AA$ \\
& Asp128:OD2-H5 & $192 \AA$ \\
ALA & Ala131 & $4.26 \AA$ \\
A:131 & Leu129 & $4.87 \AA$ \\
& Leu129 & $5.01 \AA$ \\
Leu103 & $5.45 \AA$
\end{tabular}

(c)

14. Interactions of ionized amantadine with APRT co-crystallized with AMP, PDB ID 1ZN8. (a) 3D display of the amantadine (shows in yellow) and AMP in binding pocket; (b) code color for interactions: in orange are shown the salt bridges, light green - van der Waals forces, mauve - alkyl hydrophobic interactions; (c) the distances

(Å) of the 1ZN8 - ionized amantadine interactions. 
The other two APRT structures co-crystallized with AMP show different interactions. Among the eight residues of $10 R E$ that interact with amantadine only two are AMP-binding residues (Val25 and Arg27) (Figure 15).

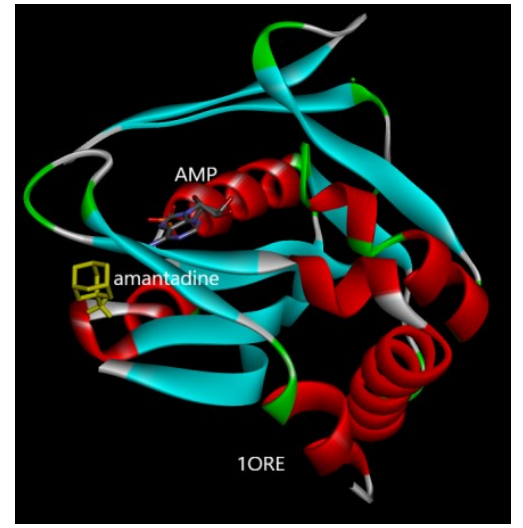

(a)

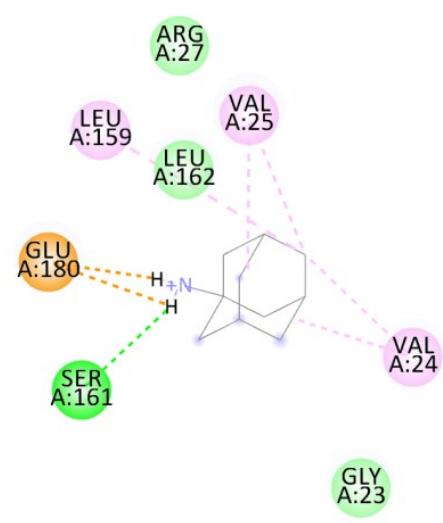

(b)

\begin{tabular}{cc}
\hline Ser161:OG-H27 & $2.04 \AA$ \\
\hline Glu180:OXT-H27 & $2.31 \AA$ \\
Glu180:O-H28 & $1.85 \AA$ \\
Val24 & $4.29 \AA$ \\
Val25 & $4.22 \AA$ \\
Val24-C9 & $4.28 \AA$ \\
Val25-C9 & $4.69 \AA$ \\
Leu159-C9 & $4.85 \AA$
\end{tabular}

(c)

Figure 15. Interactions of ionized amantadine with APRT co-crystallized with AMP, PDB ID 1ORE. (a) 3D display of the amantadine (shows in yellow) and AMP in binding pocket; (b) code color for interactions: in orange are shown the salt bridges, light green - van der Waals forces, mauve - alkyl hydrophobic interactions; (c) the distances $(\AA ̊)$ of the $10 R E$ - ionized amantadine interactions.

The molecular docking of amantadine with $4 X 44$ results in similar interactions with 1ORE. There are three AMP-binding residues that bind amantadine (Val25, Arg27, and Leu159) (Figure 16).

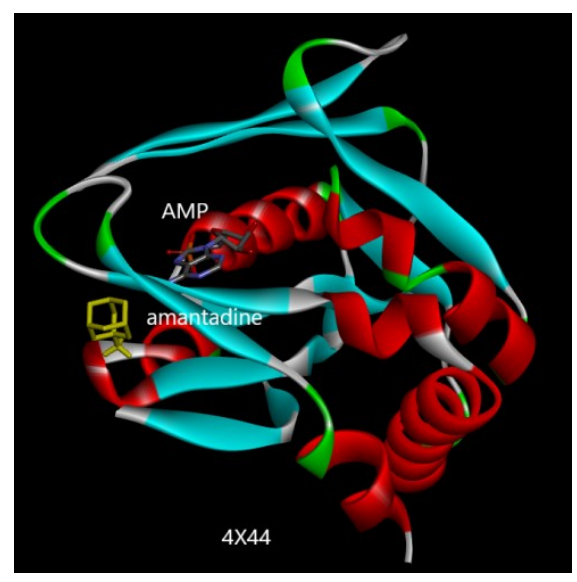

(a)

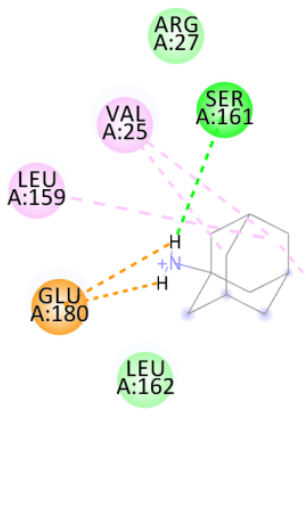

(b)

\begin{tabular}{ccc}
\hline & Ser161:OG-H5 & $2.33 \AA$ \\
\hline & Glu180:OXT-H5 & $2.29 \AA$ \\
& Glu180:O-H27 & $1.90 \AA$ \\
& Val24 & $4.81 \AA$ \\
& Val25 & $4.78 \AA$ \\
\hline VAL & Val25-C10 & $3.68 \AA$ \\
A:24 & Leu159 & $4.86 \AA$ \\
\hline & &
\end{tabular}

(c)

Figure 16. Interactions of ionized amantadine with APRT co-crystallized with AMP, PDB ID 4X44. (a) 3D display of the amantadine (shows in yellow) and AMP in binding pocket; (b) code color for interactions: in orange are shown the salt bridges, light green - van der Waals forces, mauve - alkyl hydrophobic interactions; (c) the distances $(\AA)$ of the $4 \mathrm{X} 44$ - ionized amantadine interactions.

The human APRT co-crystallized with adenine (PDB ID(s) 6FCl and 1ZN7) allows a deeper view of the amantadine interactions with this enzyme. The two structures have different behavior when docked with amantadine. Thus, among ten residues of the $6 \mathrm{FCl}$ that interact with amantadine, five bind adenine (Glu104, Arg67, Leu129, Tyr105, and Ala131) (Figure 17). 


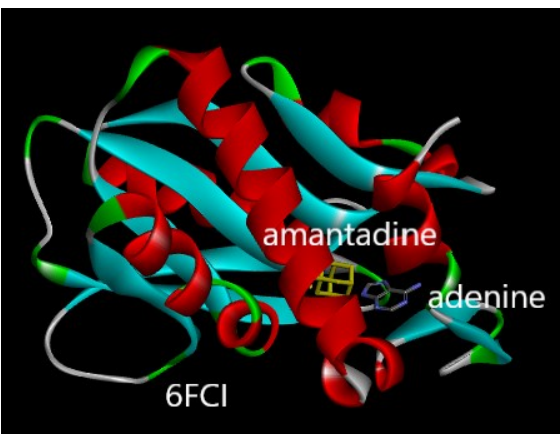

(a)

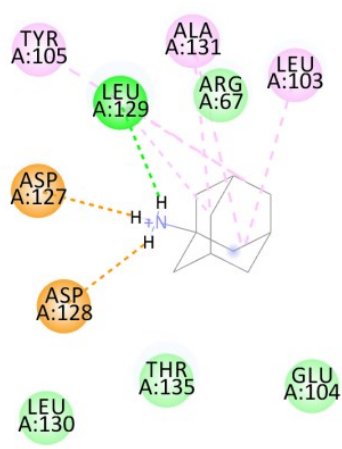

(b)

\begin{tabular}{cc}
\hline Leu129:O-H5 & $2.05 \AA$ \\
\hline Asp127-OD1 & $1.75 \AA$ \\
Asp128:OD2 & $1.81 \AA$ \\
Ala131 & $5.09 \AA$ \\
Ala131-C10 & $3.35 \AA$ \\
Ala129 & $4.85 \AA$ \\
Leu103 & $4.29 \AA$ \\
Leu129-C10 & $4.47 \AA$ \\
Tyr105 & $5.16 \AA$
\end{tabular}

(c)

Figure 17. Interactions of ionized amantadine with APRT co-crystallized with adenine, PDB ID 6FCl. (a) 3D display of the amantadine (shows in yellow) and adenine in binding pocket; (b) code color for interactions: in orange are shown the salt bridges, light green - van der Waals forces, mauve - alkyl hydrophobic interactions;

(c) the distances $(\AA)$ of the $6 \mathrm{FCl}$ - ionized amantadine interactions.

Contrary, in the case of 1ZN7 structure, molecular docking of amantadine involves different residues than adenine (Figure 18). The last two structures (PDB ID(s) 6FCl and 1ZN7), being co-crystallized with adenine, not with the substrate AMP, should be interpreted accordingly.

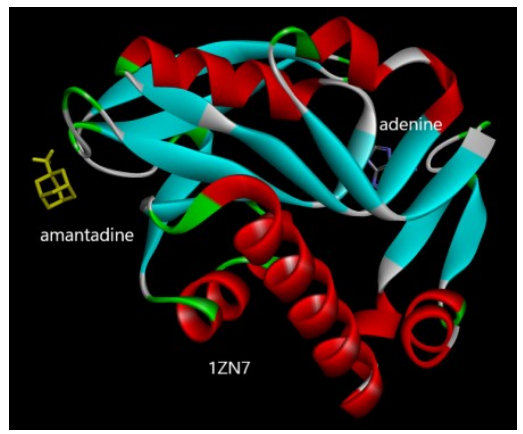

(a)

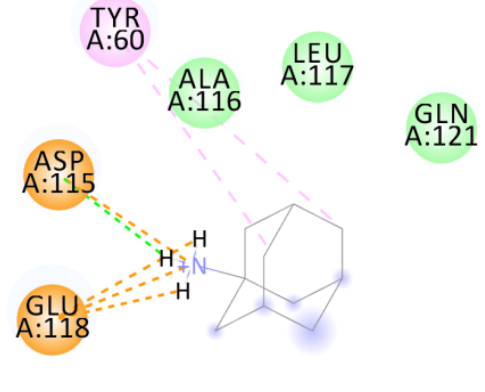

(b)

\begin{tabular}{cc}
\hline Asp115:O-H27 & $1.83 \AA$ \\
\hline Glu118:OE1-H27 & $2.03 \AA$ \\
\hline Glu118:OE1-H5 & $2.15 \AA$ \\
\hline Asp115:OD2-N1 & $5.51 \AA$ \\
\hline Glu118:OE1-N1 & $4.30 \AA$ \\
\hline Tyr60 & $4.30 \AA$ \\
Tyr60-C9 & $5.28 \AA$
\end{tabular}

(c)

Figure 18. Interactions of ionized amantadine with APRT co-crystallized with adenine, PDB ID 1ZN7. (a) 3D display of the amantadine (shows in yellow) and adenine in binding pocket; (b) code color for interactions: in orange are shown the salt bridges, light green - van der Waals forces, mauve - alkyl hydrophobic interactions;

(c) the distances $(\AA)$ of the 1 ZN7 - ionized amantadine interactions.

Molecular docking of the human ecto-5'-nucleotidase (NT5E) with ionized amantadine and the redocking with the substrates

The ecto-5'-nucleotidase (NT5E) (E.C. 3.1.3.5) catalyzes the hydrolysis of extracellular AMP to adenosine. All human NT5Es found in PDB were analyzed about the amantadine interactions. First, the redock of the substrates co-crystallized in the X-ray 3D structures was made, then amantadine ionized form molecular docking (Table 3). In all NT5Es structures analyzed, the amanatdine binds other residues than the substrate-binding region (data not shown).

Molecular docking of the human ectonucleoside triphosphate diphosphohydrolase 1 with ionized amantadine and the redocking with the substrates

The ectonucleoside triphosphate diphosphohydrolase 1 (ENTPD1) (E.C. 3.6.1.5) catalyzes the reaction: a ribonucleoside $5^{\prime}$-triphosphate $+2 \mathrm{H}_{2} \mathrm{O}=$ a ribonucleoside $5^{\prime}$-triphosphate $+2 \mathrm{H}^{+}+2$ phosphate. Because there are no matching PDB entries for human ENTDP1, were analyzed the ENTDP1 from Rattus norvegicus. 
Although the interpretation of the molecular docking of amantadine is not reliable, because the crystal structures are not with the substrates, it is worth noticing that the molecular docking of the 3ZX2 with amantadine results in an interaction with the active-site residue Glu174 by two salt bridge (Table 4).

Table 3. The energy binding $(\Delta G)$ and inhibition constant $(\mathrm{Ki})$ of the best conformation of the complex NT5E - amantadine and after redocking.

\begin{tabular}{ccccc}
\hline & \multicolumn{2}{c}{ Amantadine } & \multicolumn{2}{c}{ redocking } \\
PDB ID & $\begin{array}{c}\boldsymbol{\Delta} \boldsymbol{G} \\
\text { (kcal/mol) }\end{array}$ & $\boldsymbol{K}_{\mathrm{i}}(\boldsymbol{\mu M})$ & $\begin{array}{c}\Delta \boldsymbol{G} \\
\text { (kcal/mol) }\end{array}$ & $\boldsymbol{K}_{\mathrm{i}}(\boldsymbol{\mu M})$ \\
\hline $4 \mathrm{H} 2 \mathrm{I}$ & -8.08 & 1.2 & -4.91 & 250.92 \\
$4 \mathrm{H} 2 \mathrm{~F}$ & -7.93 & 1.55 & -6.95 & 8.01 \\
$4 \mathrm{H} 2 \mathrm{G}$ & -7.59 & 2.72 & -5.97 & 42.08 \\
$4 \mathrm{H} 1 \mathrm{~S}$ & -7.12 & 6.08 & -5.56 & 84.56 \\
\hline
\end{tabular}

${ }^{1}$ redocking with the substrates co-crystallized in PDB X-ray 3D structures.

Table 4. The energy binding $(\Delta G)$ and inhibition constant (Ki) of the best conformation of the complex ENTPD1 - amantadine.

\begin{tabular}{|c|c|c|c|}
\hline \multirow[b]{2}{*}{ PDB ID } & \multicolumn{2}{|c|}{ Amantadine } & \multirow[t]{2}{*}{ redocking } \\
\hline & $\begin{array}{c}\Delta G \\
\text { (kcal/mol) }\end{array}$ & $K_{\mathrm{i}}(\mu \mathrm{M})$ & \\
\hline $3 Z X 0$ & -6.6 & 14.56 & \multirow{3}{*}{ Not the case } \\
\hline $3 Z X 2$ & -6.45 & 18.71 & \\
\hline $3 Z X 3$ & -7.24 & 4.94 & \\
\hline
\end{tabular}

Molecular docking of the human nucleoside diphosphate kinase 3 (NDK3) with ionized amantadine and the redocking with the substrates

The nucleoside diphosphate kinase 3 (NDK3) (E.C. 2.7.4.6) catalysis the reaction: a 2'deoxyribonucleoside $5^{\prime}$-diphosphate + ATP $=$ a $2^{\prime}$-deoxyribonucleoside $5^{\prime}$-triphosphate + ADP. The human found in PDB were analyzed about the amantadine interactions (Table 5). First, the redock of the substrates co-crystallized in the X-ray 3D structures were made for three structures (PDB ID(s) 1ZS6, 2HVD, and $3 \mathrm{BBB})$. In the case of $1 \mathrm{ZS} 6$ and $3 \mathrm{BBB}$, no significant interactions of the amantadine with ATP-binding residues or with the histidine from the active site were observed. The 2HVD and 1JXV form van der Waal forces with histidine 118 from the active site (Pros-phosphohistidine intermediate) but no interactions with the ATP-binding residues.

Table 5. The energy binding $(\Delta \mathrm{G})$ and inhibition constant (Ki) of the best conformation of the complex NDK3 amantadine and after redocking.

\begin{tabular}{ccccc}
\hline & \multicolumn{2}{c}{ Amantadine } & \multicolumn{2}{c}{ redocking } \\
PDB ID & $\begin{array}{c}\Delta \boldsymbol{G} \\
(\mathrm{kcal} / \mathrm{mol})\end{array}$ & $\boldsymbol{K}_{\mathrm{i}}(\mu \mathrm{M})$ & $\begin{array}{c}\Delta \boldsymbol{G} \\
(\mathbf{k c a l} / \mathbf{m o l})\end{array}$ & $\boldsymbol{K}_{\mathrm{i}}(\mu \mathrm{M})$ \\
\hline 1ZS6 & -7.34 & 4.15 & -5.96 & 42.42 \\
2HVD & -6.55 & 15.69 & -5.81 & 54.99 \\
1JXV & -7.13 & 5.97 & \multicolumn{2}{c}{ not the case } \\
3BBB & -5.63 & 74.71 & $-5.88^{2}$ & $49.34^{2}$ \\
& & & $-5.35^{3}$ & $119.96^{3}$ \\
\hline
\end{tabular}

${ }^{1}$ redocking with the substrates co-crystallized in PDB X-ray 3D structures; ${ }^{2}$ redock of 2 -deoxyadenosine- 5 'monophosphate; ${ }^{3}$ redock of 2 '-deoxyguanosine-5'-monophosphate.

Molecular docking of the human purine nucleoside phosphorylase 1 (PNP1) with ionized amantadine and the redocking with the substrates

The human purine nucleoside phosphorylase 1 (PNP1) (E.C. 2.4.2.1) catalyses the reaction: a purine D- 
ribonucleotide + phosphate $=$ a purine nucleobase + alpha-D-ribose 1-phosphate. The reaction is part of purine nucleoside salvage (UniProtKB P00491). The human PNP1s retrieved from the PDB were analyzed about the amantadine interactions (Table 6). The molecular docking of the PNP1 structures shows no relevant interactions with amantadine. Some of the PNP1 interact with amantadine with only one bindingsite residue - the 1ULB interacts with Asn243 and the 1RFG forms a salt bridge with Glu201. The 2AOW, 2AOX, 2AOY, 1RSZ form van der Waals interactions with only one binding-site residue - GIn201.

Table 6. The energy binding $(\Delta \mathrm{G})$ and inhibition constant (Ki) of the best conformation of the complex PNP1 - amantadine and after redocking.

\begin{tabular}{ccccc}
\hline & \multicolumn{2}{c}{ Amantadine } & \multicolumn{2}{c}{ redocking } \\
PDB ID & $\begin{array}{c}\boldsymbol{\Delta} \boldsymbol{G} \\
\text { (kcal/mol) }\end{array}$ & $\boldsymbol{K}_{\mathrm{i}}(\boldsymbol{\mu} \mathrm{M})$ & $\begin{array}{c}\boldsymbol{\Delta} \boldsymbol{G} \\
\text { (Kcal/mol) }\end{array}$ & \multicolumn{2}{c}{$\boldsymbol{K}_{\mathbf{i}}$} \\
\hline 1ULA & -7.31 & 4.35 & \multicolumn{2}{c}{ not the case } \\
1ULB & -6.74 & 11.47 & -5.61 & $77.85 \mu \mathrm{M}$ \\
2AOW & -6.52 & 16.57 & -8.63 & $473.78 \mathrm{nM}$ \\
2AOX & -6.5 & 17.29 & -8.69 & $425.71 \mathrm{nM}$ \\
2AOY & -6.46 & 18.38 & -8.89 & $306.03 \mathrm{nM}$ \\
1RSZ & -6.41 & 19.92 & -8.35 & $760.14 \mathrm{nM}$ \\
1RFG & -6.4 & 20.45 & -7.42 & $3.67 \mu \mathrm{M}$ \\
\hline
\end{tabular}

Molecular docking of the creatine kinases (CKs) with ionized amantadine and redocking

The CK (E.C. 2.7.3.2) catalyses the reversible reaction: ATP + creatine $=\mathrm{ADP}+\mathrm{H}^{+}+\mathrm{N}$-phosphocreatine. The different CKs type (UniProtKB P11009, P17540, P00563, P06732, P12277, P05122, and Q9XSC6) retieved form the PDB were analysed about the amantadine interactions (Table 7). Molecular docking results show no interactions of amantadine with ATP-binding residues or nucleotide (ATP) - binding regions.

Table 7. The energy binding $(\Delta \mathrm{G})$ and inhibition constant (Ki) of the best conformation of the complex CK - amantadine and after redocking.

\begin{tabular}{|c|c|c|c|c|}
\hline \multirow[b]{2}{*}{ PDB ID } & \multicolumn{2}{|c|}{ Amantadine } & \multicolumn{2}{|c|}{ redocking $^{1}$} \\
\hline & $\begin{array}{c}\Delta G \\
(\mathrm{kcal} / \mathrm{mol})\end{array}$ & $K_{\mathrm{i}}$ & $\Delta G(\mathrm{Kcal} / \mathrm{mol})$ & $K_{\mathrm{i}}$ \\
\hline \multicolumn{5}{|c|}{ mitochondrial CK } \\
\hline 1CRK & -6.65 & $13.26 \mu \mathrm{M}$ & \multicolumn{2}{|c|}{ not the case } \\
\hline $4 Z 9 M$ & -7.3 & $4.49 \mu \mathrm{M}$ & -8.64 & $462.56 \mathrm{nM}$ \\
\hline \multicolumn{5}{|c|}{ muscle CK } \\
\hline 2CRK & -7.65 & $2.45 \mu \mathrm{M}$ & \multirow{2}{*}{\multicolumn{2}{|c|}{$\begin{array}{l}\text { not the case } \\
\text { not the case }\end{array}$}} \\
\hline $1 \mathrm{IOE}$ & -5.81 & $54.93 \mu \mathrm{M}$ & & \\
\hline $1 U 6 R$ & -6.37 & $21.24 \mu \mathrm{M}$ & -7.84 & $1.78 \mu \mathrm{M}$ \\
\hline \multicolumn{5}{|l|}{ brain $\mathrm{CK}$} \\
\hline 3DRB & -6.39 & $20.63 \mu \mathrm{M}$ & \multicolumn{2}{|c|}{ not the case } \\
\hline 3DRE & -6.99 & $7.57 \mu \mathrm{M}$ & \multicolumn{2}{|c|}{ not the case } \\
\hline $1 \mathrm{QH} 4$ & -7.45 & $3.44 \mu \mathrm{M}$ & \multicolumn{2}{|c|}{ not the case } \\
\hline \multicolumn{5}{|c|}{ retinal CK } \\
\hline 1GOW & -7.12 & $6.07 \mu \mathrm{M}$ & \multicolumn{2}{|c|}{ not the case } \\
\hline
\end{tabular}

\section{Discussion}

The PD is an idiopathic, progressive disease of the central neurological system. The treatment of dyskinesia remains a critical issue in the PD treatment strategy, levodopa-induced dyskinesia being a real challenge for both patients and healthcare professionals. The combination of levodopa - amantadine is useful for the people who do not tolerate optimal doses of levodopa and allows better control of the 
disease. Among other biomarkers modified in PD, there is a dysregulation of some human AK isoforms that are also altered in other muscle diseases. The AK1 and AK2 isoforms are also involved in modulation immunity and inflammation in many diseases. These observations formed the basis of the design of this study.

Because AK is an important enzyme for energetic metabolism, its involvement in a variety of diseases is not surprising. Because the ATP is the most used "exchange currency" in nature, the crucial role of AK is energetic metabolism explains the interest in the study of this enzyme. Nine human AKs isoforms have been identified, each with important characteristics that differentiate them [60]. Five AKs isoforms have the X-ray structure deposited in public databases but only four isoforms are in closed-conformation (AK1, $A K 2$, AK4, and AK5). Consequently, only the AKs in closed-conformation were selected for the present study - five human AKs, one AK1 from zebrafish, and two bacterial AKs.

Experimental studies are very often costly and time-consuming, so conducting an experimental study without strong theoretical research is not a pragmatic approach. Molecular docking is a useful method for first analyzes of new protein-ligand interaction. This method allowed analysis of the selective binding of amantadine to the AKs' isoforms implicated in PD. The $\Delta G$ and Ki data provide only a first view of the amantadine binding to different AKs. Thus, a deep analysis of the amantadine with the AKs residues illustrated that there are notable differences between all human AKs investigated. It was previously described a down-regulation of the mitochondrial isoforms AK2 and AK4 in the stages 3-6 and an upregulation of the cytosolic isoform AK1 in the stages 5-6, as compensation of altered purine metabolism (Figure 19) [24]. There are well-known motor deficits in PD - slowness of movement, muscle rigidity, and tremor at rest, the tremor that decreases when the body is actively engaged in purposeful activity [61][63]. The cytosolic AK1 and the mitochondrial AK2 are dysregulated in different muscle pathology. In contrast with PD, in Duchenne muscular dystrophy - an X-linked recessive disease - there is a progressive weakness mainly of the pelvic girdle and shoulder girdle, pseudohypertrophy due to the infiltration by fat and connective tissue with immobilization at young ages and cardiomyopathy [64], [65]. The role of AK1 in inflammation has been demonstrated, but its role in maintaining neuroinflammation is not yet known (Figure 19) [66].

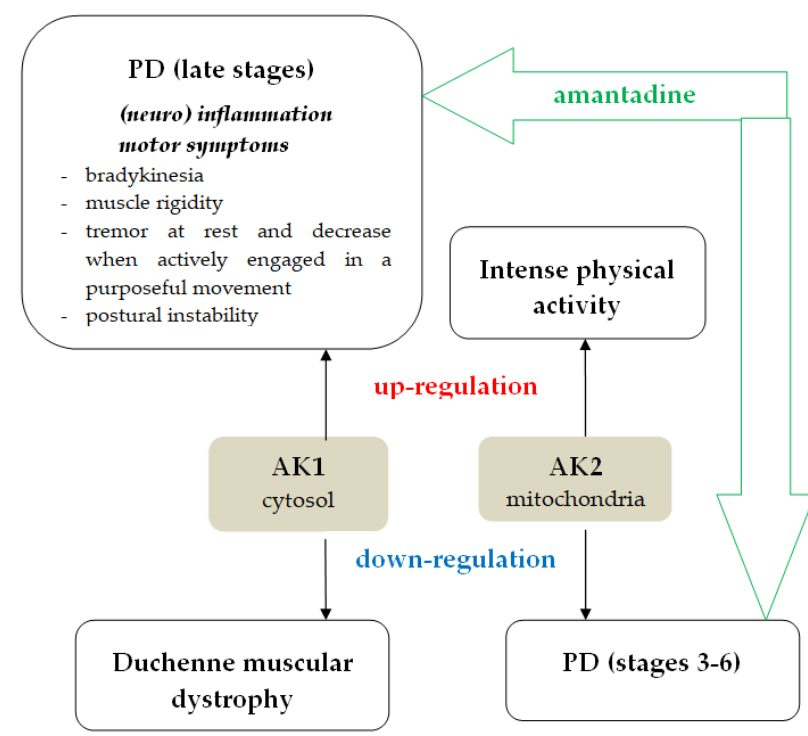

Figure 19. The involvement of human AK1 and human AK2 in PD, inflammation, and muscle activity. The amantadine treatment efficacy in PD developed the hypothesis that amantadine - AK interactions. 
According to the scientific literature, there is no connection between the amantadine and AKs in PD. However, because the mechanism of amantadine in PD is not yet known, the investigation of the possible mechanism of amantadine is of great interest. The present study was conducted with the premise that some markers up-regulated or down-regulated in PD could be the target for amantadine. Many studies described the association of some clinical symptoms with dysregulation of different enzymes. The observation that many AKs isoforms are altered in PD leads to the hypothesis that there is a connection between amantadine and these AKs isoforms. Also, other enzymes already described to be dysregulated in PD was investigated - APRT, NT5E, ENTPD1, NDK3, and PNP1 [24]. More, CK was included in the study because of its proven involvement in some muscle diseases [27].

Our experiments show that the human cytosolic AK1 interacts mainly with ionized amantadine by residues from the AMP binding region. Even if the two human AK1s analyzed do not form the same interactions with amantadine, the observation could be aware of the efficacy of the amantadine in the late stages of PD, because, as mentioned earlier, AK1 activity could compensate for purine metabolism dysfunctions. Regarding AK1 from other species, the zebrafish AK1 interactions with amantadine are more similar to those of the AK from B.stearothermophilus. Moreover, the interactions with ionized amantadine are different for the three AK1 enzymes analyzed. Regarding the mitochondrial human AK2, the interactions with ionized amantadine are most similar to AK1 (PDB ID 1Z83) with five residues in common Thr44, Val74, Phe101, Arg103, and Gln107. Thus, the results of the present study highlight the relevance of $A K 1$ and $A K 2$ in PD and provide a valid perspective for further investigation of the role of amantadine in inhibiting these enzymes. The connection between cytosolic and mitochondrial human AKs was extensively studied by Dzeja et al. who made a very clear demonstration of the type "bucket-brigade" process by which AK1 and AK2 isoforms facilitate the transfer of the enzyme substrates without changes in the metabolite concentration in the cellular compartments [67].

It is not possible to establish the mechanism of action of amantadine in PD observing only the interaction of the drug with AKs, but the results of the present study provide a valuable perspective for understanding the pathogenesis of PD. So the next step was to analyze of a large group of proteins. Among the enzymes analyzed for their affinity for amantadine, APRT shows remarkable results. Because there are available many APRTs X-ray 3D structures co-crystallized with AMP and with adenine, we were able to compare the molecular docking results. The molecular docking of amantadine ionized form with APRTs demonstrated that amantadine interacts with AMP-binding residues. Although the structures of APRTs co-crystallized with adenine shows identical residues that bind amantadine and adenine, there are some differences between the APRTs structures tested. Contrary, the results obtained by molecular docking of amantadine with APRTs X-ray 3D structures co-crystallized with AMP strongly indicated that amantadine could target adenine metabolism.

Here, a preliminary analysis of some key factors described in PD pathogenesis reveals that there are at least two enzymes involved in adenine metabolism that worth further investigation - AK and APRT. Then, before an experimental confirmation of the molecular docking results, a molecular dynamics simulation study aid to observe the proper binding of amantadine.

The present study aimed to try to make sense of the anti-parkinsonian effects of the amantadine. The methods used in the present study do not elucidate the mechanism of amantadine, but demonstrate that in PD there is a link between amantadine and some enzymes described to be modified in different stages of the disease. 


\section{Conclusions}

The PD is a progressive neurodegenerative disease characterized mainly by motility impairment. There is no curative treatment but there are some therapeutic strategies for the treatment of dyskinesia, amantadine being used until now even for better control of levodopa-induced dyskinesia. Also, there is demonstrated that in the clinical evolution of PD, many enzymes are modified - some AK isoforms, APRT, NT5E, ENTPD1, NDK3, and PNP1. Using available X-ray 3D structures of these enzymes or their counterparts from other organisms, two enzymes show relevant results - AK and APRT. The closedconformation of the AK is most reliable due to the catalytic particularities of the enzyme - the movement of the domains is coordinated by the sequential addition of the substrates. The molecular docking experiments demonstrated that human AK1 and AK2 with ionized amantadine by AMP binding residues but there are notable differences between the two AK isoforms. Other AKs included in the study, further demonstrated the specific interaction of amantadine with human AKs. The study of amantadine interaction with different AKs' isoforms has more to give. The studies of the PD highlighted that there is a relationship between the key factors involved in PD. Among them, mitochondrial factors have a crucial role in the disease pathogenesis. The APRT structures co-crystallized with AMP results in the best interactions with amantadine, the AMP-binding residues form strong interactions with amantadine. The results of the present study offer a new perspective for further investigation of the connections between amantadine treatment of PD and some enzymes involved in purine metabolism.

Funding: This work was supported by a grant of Ministry of Research and Innovation, CNCS - UEFISCDI, project number PN-IIIP4-ID-PCCF-2016-0016, within PNCDI III and by a mobility project of the Romanian Ministry of Research and Innovation, CNCS - UEFISCDI, the project number PN-III-P1-1.1-MCD-2018-0108, within PNCDI III.

Acknowledgements: This work is dedicated to the memory of Professor Dr. Octavian Bârzu, my mentor in the study of nucleoside monophosphate kinases. I would like to thank Tudor Constantin Badea for his generous advice during the mobility project. Many thanks to the reviewers for their generous comments.

Conflicts of Interest: The authors declare no conflict of interest. The funders had no role in the design of the study; in the collection, analyses, or interpretation of data; in the writing of the manuscript, or in the decision to publish the results.

\section{References}

[1] D.J. Gelb, E. Oliver, S. Gilman. Diagnostic criteria for Parkinson disease. Arch. Neurol. 56 (1999) 3339.

[2] J. Jankovic. Parkinson's disease: clinical features and diagnosis. J. Neurol. Neurosurg. Psychiatry 79 (2008) 368-376.

[3] F. Sun, Y. Deng, X. Han, Q. Liu, P. Zhang, R. Manzoor, H. Ma. A secret that underlies Parkinson's disease: The damaging cycle. Neurochem. Int. 129 (2019) 104484.

[4] B. Boland, F.M. Platt. Bridging the age spectrum of neurodegenerative storage diseases. Best Pract. Res. Clin. Endocrinol. Metab. 29 (2015) 127-143

[5] D. Elstein, R. Alcalay, A. Zimran. The emergence of Parkinson disease among patients with Gaucher disease. Best Pract. Res. Clin. Endocrinol. Metab. 29 (2015) 249-259.

[6] E. Bezard, C.E. Gross, M.C. Fournier, S. Dovero, B. Bloch, M. Jaber. Absence of MPTP-induced neuronal death in mice lacking the dopamine transporter. Exp. Neurol. 155 (1999) 268-273.

[7] Z.R. Zhang, X.R. Zhang, X.Q. Luan, X.S. Wang, W.W. Wang, X.Y. Wang, B. Shao, C.L. Xie. Striatal overexpression of $\beta$-arrestin2 counteracts L-dopa-induced dyskinesia in 6-hydroxydopamine lesioned Parkinson's disease rats. Neurochem. Int. 131 (2019) 104543. 
[8] G. Orlando, A. Chiavaroli, S. Leone, L. Brunetti, M. Politi, L. Menghini, L. Recinella, C. Ferrante. Inhibitory Effects Induced by Vicia faba, Uncaria rhyncophylla, and Glycyrrhiza glabra Water Extracts on Oxidative Stress Biomarkers and Dopamine Turnover in HypoE22 Cells and Isolated Rat Striatum Challenged with 6-Hydroxydopamine. Antioxidants (Basel) 8 (2019) E602.

[9] V. Leta, P. Jenner, K.R. Chaudhuri, A. Antonini. Can therapeutic strategies prevent and manage dyskinesia in Parkinson's disease? An update. Expert Opin. Drug Saf. 18 (2019) 1203-1218.

[10] B.S. Connolly, A.E. Lang. Pharmacological treatment of Parkinson disease: a review. JAMA 311 (2014) 1670-1683.

[11] W. Oertel, J.B. Schulz. Current and experimental treatments of Parkinson disease: A guide for neuroscientists. J. Neurochem. 139 Suppl 1 (2016) 325-337.

[12] H.H. Liu, N.C. Yeh, Y.F. Wu, Y.R. Yang, R.Y. Wang, F.Y. Cheng. Effects of Tai Chi Exercise on Reducing Falls and Improving Balance Performance in Parkinson's Disease: A Meta-Analysis. Parkinsons Dis. 2019 (2019) 9626934.

[13] S. Vasudevan, J. Kajtez, A.I. Bunea, A. Gonzalez-Ramos, T. Ramos-Moreno, A. Heiskanen, M. Kokaia, N.B. Larsen, A. Martínez-Serrano, S.S. Keller, J. Emnéus. Leaky Optoelectrical Fiber for Optogenetic Stimulation and Electrochemical Detection of Dopamine Exocytosis from Human Dopaminergic Neurons. Adv. Sci. (Weinh) 6 (2019) 1902011.

[14] N.G.M. Oonk, K.L.L. Movig, E.M. Munster, K. Koehorst-Ter Huurne, J. van der Palen, L.D.A. Dorresteijn. The effect of a structured medication review on quality of life in Parkinson's disease: The study protocol. Contemp. Clin. Trials Commun. 13 (2019) 100308.

[15] E.K. Perry, M. Curtis, D.J. Dick, J.M. Candy, J.R. Atack, C.A. Bloxham, G. Blessed, A. Fairbairn, B.E. Tomlinson, R.H. Perry. Cholinergic correlates of cognitive impairment in Parkinson's disease: comparisons with Alzheimer's disease. J. Neurol. Neurosurg. Psychiatry 48 (1985) 413-421.

[16] J. Glowinski. Recent finding on dopaminergic transmission in the basal ganglia. Adv. Neurol. 53 (1990) 67-73.

[17] J.P. McEvoy. FDA-Approved Medications to Treat Tardive Dyskinesia. J. Clin. Psychiatry 81 (2019) NU18041BR3C.

[18] C. Zhuo, X. Zhu, R. Jiang, F. Ji, Z. Su, R. Xue, Y. Zhou. Comparison for Efficacy and Tolerability among Ten Drugs for Treatment of Parkinson's Disease: A Network Meta-Analysis. Sci. Rep. 8 (2017) 45865.

[19] K. Nishiyama, M. Sugishita, H. Kurisaki, M. Sakuta. Reversible memory disturbance and intelligence impairment induced by long-term anticholinergic therapy. Intern. Med. 37 (1998) 514-518.

[20] Y. Baba, M.A. Higuchi, H. Abe, K. Fukuyama, R. Onozawa, Y. Uehara, T. Inoue, T. Yamada. Anticholinergics for axial symptoms in Parkinson's disease after subthalamic stimulation. Clin. Neurol. Neurosurg. 114 (2012) 1308-1311.

[21] A. Djamshidian, W. Poewe. Apomorphine and levodopa in Parkinson's disease: Two revolutionary drugs from the 1950's. Parkinsonism Relat. Disord. 33 Suppl 1 (2016) S9-S12.

[22] P. Turcano, M.M. Mielke, J.H. Bower, J.E. Parisi, J.K. Cutsforth-Gregory, J.E. Ahlskog, R. Savica. Levodopa-induced dyskinesia in Parkinson disease: A population-based cohort study. Neurology 91 (2018) e2238-e2243.

[23] K. Dashtipour, A.R. Tafreshi, R. Pahwa, K.E. Lyons. Extended-Release Amantadine for LevodopaInduced Dyskinesia. Expert Rev. Neurother. 19 (2019) 293-299.

[24] P. Garcia-Esparcia, K. Hernández-Ortega, B. Ansoleaga, M. Carmona, I. Ferrer. Purine metabolism gene deregulation in Parkinson's disease. Neuropathol. Appl. Neurobiol. 41 (2015) 926-940.

[25] T.M. Seccia, A. Atlante, V. Vulpis, E. Marra, S. Passarella, A. Pirrelli. Mitochondrial energy metabolism in the left ventricular tissue of spontaneously hypertensive rats: abnormalities in both adeninenucleotide and phosphate translocators and enzyme adenylate-kinase and creatinephosphokinase activities. Clin. Exp. Hypertens. 20 (1998) 345-358. 
[26] Z.H. Wang, X.L. Cai, L. Wu, Z. Yu, J.L. Liu, Z.N. Zhou, J. Liu, H.T. Yang. Mitochondrial energy metabolism plays a critical role in the cardioprotection afforded by intermittent hypobaric hypoxia. Exp. Physiol. 97 (2012) 1105-1118.

[27] Y. Ge, M.P. Molloy, J.S. Chamberlain, P.C. Andrews. Proteomic analysis of mdx skeletal muscle: Great reduction of adenylate kinase 1 expression and enzymatic activity. Proteomics 3 (2003) 1895-1903.

[28] T. Gamberi, T. Fiaschi, E. Valocchia, A. Modesti, P. Mantuano, J.F. Rolland, F. Sanarica, A. De Luca, F. Magherini. Proteome analysis in dystrophic $\mathrm{mdx}$ mouse muscle reveals a drastic alteration of key metabolic and contractile proteins after chronic exercise and the potential modulation by antioxidant compounds. J. Proteomics 170 (2018) 43-58.

[29] T. Fröhlich, B. Reitter, D. Scheffner, R.H. Schirmer, R. Untucht-Grau. Muscle adenylate kinase in Duchenne muscular dystrophy. Biochim. Biophys. Acta 883 (1986) 598-603.

[30] M. Hamada, H. Okuda, K. Oka, T. Watanabe, K. Ueda, M. Nojima, S.A. Kuby, M. Manship, F.H. Tyler, F.A. Ziter. An aberrant adenylate kinase isoenzyme from the serum of patients with Duchenne muscular dystrophy. Biochim. Biophys. Acta 660 (1981) 227-237.

[31] R. Spector. Transport of amantadine and rimantadine through the blood-brain barrier. J. Pharmacol. Exp. Ther. 244 (1988) 516-519.

[32] S. Moon, J. Kim, E. Bae. Structural analyses of adenylate kinases from Antarctic and tropical fishes for understanding cold adaptation of enzymes. Sci. Rep. 7 (2017) 16027.

[33] T.P. Schrank, D.W. Bolen, V.J. Hilser. Rational modulation of conformational fluctuations in adenylate kinase reveals a local unfolding mechanism for allostery and functional adaptation in proteins. Proc. Natl. Acad. Sci. U.S.A. 106 (2009) 16984-16989.

[34] M.B. Berry, G.N. Phillips. Crystal structures of Bacillus stearothermophilus adenylate kinase with bound Ap5A, Mg2+ Ap5A, and Mn2+ Ap5A reveal an intermediate lid position and six coordinate octahedral geometry for bound Mg2+ and Mn2+. Proteins 32 (1998) 276-288

[35] M. Silva, C.H. Silva, J. lulek, O.H. Thiemann. Three-dimensional structure of human adenine phosphoribosyltransferase and its relation to DHA-urolithiasis. Biochemistry 43 (2004) 7663-7671.

[36] C.H. Silva, M. Silva, J. lulek, O.H. Thiemann. Structural complexes of human adenine phosphoribosyltransferase reveal novel features of the APRT catalytic mechanism. J. Biomol. Struct. Dyn. 25 (2008) 589-597.

[37] J. Huyet, M. Ozeir, M.C. Burgevin, B. Pinson, F. Chesney, J.M. Remy, A.R. Siddiqi, R. Lupoli, G. Pinon, C. Saint-Marc, J.F. Gibert, R. Morales, I. Ceballos-Picot, R. Barouki, B. Daignan-Fornier, A. OlivierBandini, F. Augé, P. Nioche. Structural Insights into the Forward and Reverse Enzymatic Reactions in Human Adenine Phosphoribosyltransferase. Cell Chem. Biol. 25 (2018) 666-676.e4.

[38] K. Knapp, M. Zebisch, J. Pippel, A. El-Tayeb, C.E. Müller, N. Sträter. Crystal structure of the human ecto-5'-nucleotidase (CD73): insights into the regulation of purinergic signaling. Structure 20 (2012) 2161-2173.

[39] D.P. Heuts, M.J. Weissenborn, R.V. Olkhov, A.M. Shaw, J. Gummadova, C. Levy, N.S. Scrutton. Crystal structure of a soluble form of human CD73 with ecto-5'-nucleotidase activity. Chembiochem 13 (2012) 2384-2391.

[40] M.F. Giraud, F. Georgescauld, I. Lascu, A. Dautant. Crystal structures of S120G mutant and wild type of human nucleoside diphosphate kinase A in complex with ADP. J. Bioenerg. Biomembr. 38 (2006) 261-264.

[41] K. Min, H.K. Song, C. Chang, S.Y. Kim, K.J. Lee, S.W. Suh. Crystal structure of human nucleoside diphosphate kinase A, a metastasis suppressor. Proteins 46 (2002) 340-342.

[42] T.S. Dexheimer, S.S. Carey, S. Zuohe, V.M. Gokhale, X. Hu, L.B. Murata, E.M. Maes, A. Weichsel, D. Sun, E.J. Meuillet, W.R. Montfort, L.H. Hurley. NM23-H2 may play an indirect role in transcriptional activation of c-myc gene expression but does not cleave the nuclease hypersensitive element III(1). Mol. Cancer Ther. 8 (2009) 1363-1377. 
[43] S.E. Ealick, Y.S. Babu, C.E. Bugg, M.D. Erion, W.G. Guida, J.A. Montgomery, J.A. Secrist. Application of $X$-ray crystallographic methods in the design of purine nucleoside phosphorylase inhibitors. Ann. $N$. Y. Acad. Sci. 685 (1993) 237-247.

[44] A.S. Murkin, M.R. Birck, A. Rinaldo-Matthis, W. Shi, E.A. Taylor, S.C. Almo, V.L. Schramm. Neighboring group participation in the transition state of human purine nucleoside phosphorylase. Biochemistry 46 (2007) 5038-5049.

[45] F. Canduri, R.G. Silva, D.M. dos Santos, M.S. Palma, L.A. Basso, D.S. Santos, W.F. de Azevedo. Structure of human PNP complexed with ligands. Acta Crystallogr. D Biol. Crystallogr. 61 (2005) 856862.

[46] K. Fritz-Wolf, T. Schnyder, T. Wallimann, W. Kabsch. Structure of mitochondrial creatine kinase. Nature 381 (1996) 341-345.

[47] J.K. Rao, G. Bujacz, A. Wlodawer. Crystal structure of rabbit muscle creatine kinase. FEBS Lett. 439 (1998) 133-137.

[48] Y.Q. Shen, L. Tang, H.M. Zhou, Z.J. Lin. Structure of human muscle creatine kinase. Acta Crystallogr. D Biol. Crystallogr. 57 (2001) 1196-1200.

[49] J.F. Ohren, M.L. Kundracik, C.L. Borders, P. Edmiston, R.E. Viola. Structural asymmetry and intersubunit communication in muscle creatine kinase. Acta Crystallogr. D Biol. Crystallogr. 63 (2007) 381-389.

[50] S.M. Bong, J.H. Moon, K.H. Nam, K.S. Lee, Y.M. Chi, K.Y. Hwang. Structural studies of human braintype creatine kinase complexed with the ADP-Mg2+-NO3- -creatine transition-state analogue complex. FEBS Lett. 582 (2008) 3959-3965.

[51] M. Eder, U. Schlattner, A. Becker, T. Wallimann, W. Kabsch, K. Fritz-Wolf. Crystal structure of braintype creatine kinase at 1.41 A resolution. Protein Sci. 8 (1999) 2258-2269.

[52] D. Tisi, B. Bax, A. Loew. The three-dimensional structure of cytosolic bovine retinal creatine kinase. Acta Crystallogr. D Biol. Crystallogr. 57 (2001) 187-193.

[53] D.S. Goodsell, A.J. Olson. Automated docking of substrates to proteins by simulated annealing. Proteins 8 (1990) 195-202.

[54] G.M. Morris, R. Huey, W. Lindstrom, M.F. Sanner, R.K. Belew, D.S. Goodsell, A.J. Olson. AutoDock4 and AutoDockTools4: Automated docking with selective receptor flexibility. J. Comput. Chem. 30 (2009) 2785-2791.

[55] S.M. Rizvi, S. Shakil, M. Haneef. A simple click by click protocol to perform docking: AutoDock 4.2 made easy for non-bioinformaticians. EXCLI J 12 (2013) 831-857.

[56] M.B. Berry, B. Meador, T. Bilderback, P. Liang, M. Glaser, G.N. Phillips. The closed conformation of a highly flexible protein: the structure of E. coli adenylate kinase with bound AMP and AMPPNP. Proteins 19 (1994) 183-198.

[57] P. Rogne, M. Rosselin, C. Grundström, C. Hedberg, U.H. Sauer, M. Wolf-Watz. Molecular mechanism of ATP versus GTP selectivity of adenylate kinase. Proc. Natl. Acad. Sci. U.S.A. 115 (2018) 3012-3017.

[58] M. Yamada, M. Sugahara, Y. Hishitani, M. Nobumoto, A. Nakazawa. Isolation and characterization of mutated mitochondrial GTP:AMP phosphotransferase. J. Mol. Biol. 280 (1998) 551-558.

[59] C. Panayiotou, N. Solaroli, M. Johansson, A. Karlsson. Evidence of an intact N-terminal translocation sequence of human mitochondrial adenylate kinase 4. Int. J. Biochem. Cell Biol. 42 (2010) 62-69.

[60] C. Panayiotou, N. Solaroli, A. Karlsson. The many isoforms of human adenylate kinases. Int. J. Biochem. Cell Biol. 49 (2014) 75-83.

[61] D.S. Andres, O. Darbin. Complex Dynamics in the Basal Ganglia: Health and Disease Beyond the Motor System. J Neuropsychiatry Clin. Neurosci. 30 (2018) 101-114.

[62] A.M. Graybiel. The basal ganglia. Curr. Biol. 10 (2000) R509-11.

[63] H. Newman, F.C. Liu, A.M. Graybiel. Dynamic ordering of early generated striatal cells destined to form the striosomal compartment of the striatum. J. Comp. Neurol. 523 (2015) 943-962.

[64] E.M. Yiu, A.J. Kornberg. Duchenne muscular dystrophy. J. Paediatr. Child Health 51 (2015) 759-764. 
[65] B. Sunitha, M. Kumar, N. Gowthami, S. Unni, N. Gayathri, T.S. Keshava Prasad, A. Nalini, K. Polavarapu, S. Vengalil, V. Preethish-Kumar, B. Padmanabhan, M.M. Srinivas Bharath. Human muscle pathology is associated with altered phosphoprotein profile of mitochondrial proteins in the skeletal muscle. J. Proteomics 211 (2020) 103556.

[66] Y. Gao, X. Zhou, H. Wang, R. Liu, Q. Ye, Q. Zhao, Z. Ming, H. Dong. Immunization with recombinant schistosome adenylate kinase 1 partially protects mice against Schistosoma japonicum infection. Parasitol. Res. 116 (2017) 1665-1674.

[67] P. Dzeja, A. Terzic. Adenylate kinase and AMP signaling networks: metabolic monitoring, signal communication and body energy sensing. Int. J. Mol. Sci. 10 (2009) 1729-1772. 
Table S1. The characteristics of the AKs included in the study.

\begin{tabular}{|c|c|c|c|c|c|c|}
\hline & $\begin{array}{l}\text { PDB } \\
\text { ID }\end{array}$ & Organism & Residues & $\begin{array}{l}\text { co-crystallized } \\
\text { with substrate or } \\
\text { analog substrate }\end{array}$ & mutations & reference \\
\hline AK1 & $2 \mathrm{C95}$ & Homo sapiens & 194 & $\mathrm{~B}_{4} \mathrm{P}^{1} ;$ malonate ion & no & unpublished \\
\hline AK1 & $1 Z 83$ & Homo sapiens & 196 & $\mathrm{Ap} 5 \mathrm{~A}^{2} ; \mathrm{SO}_{4}{ }^{2-} ; \mathrm{Zn}^{2+}$ & no & unpublished \\
\hline AK1 & $5 X Z 2$ & Danio rerio & 196 & $\mathrm{Ap} 5 \mathrm{~A} ; \mathrm{SO}_{4}^{2-}$ & no & {$[32]$} \\
\hline AK2 & $2 \mathrm{CgY}$ & Homo sapiens & 242 & B4P; 1,2-ethandiol & no & unpublished \\
\hline AK4 & $2 A R 7$ & Homo sapiens & 246 & no & no & unpublished \\
\hline AK4 & 2BBW & Homo sapiens & 246 & $\mathrm{GP}^{3}$ & no & unpublished \\
\hline \multirow[t]{3}{*}{ AK5 } & 2BWJ & Homo sapiens & 199 & AMP & R135M & unpublished \\
\hline & $3 \mathrm{HPQ}$ & E.coli & 214 & Ap5A & no & [33] \\
\hline & $1 \mathrm{ZIP}$ & B. stearothermophilus & & Ap5A; $\mathrm{Mn}^{2+} ; \mathrm{Zn}^{2+}$ & no & [34] \\
\hline
\end{tabular}

${ }^{1}$ P1, P4-Di(adenosine)tetraphosphate, ${ }^{2}$ Bis(adenosine)-5'-pentaphosphate, ${ }^{3}$ diguanosine-pentaphosphate.

Table S2. The characteristics of the APRTs included in the study.

\begin{tabular}{|c|c|c|c|c|c|}
\hline PDB ID & Organism & Residues & $\begin{array}{c}\text { co-crystallized } \\
\text { with substrate or } \\
\text { analog substrate }\end{array}$ & mutations & reference \\
\hline 1ORE & Homo sapiens & 180 & $\mathrm{AMP}^{1} ; \mathrm{Cl}^{-}$ & no & [35] \\
\hline $1 Z N 7$ & Homo sapiens & 180 & $\begin{array}{c}\mathrm{PRP}^{2} ; \mathrm{HSX}^{3}, \mathrm{ADE}^{4} ; \\
\mathrm{PO}_{4}^{-} ; \mathrm{Mg}_{2}^{+}\end{array}$ & no & [36] \\
\hline $1 Z N 8$ & Homo sapiens & 180 & $\mathrm{AMP} ; \mathrm{Cl}^{-}$ & no & [36] \\
\hline $1 Z N 9$ & Homo sapiens & 180 & AMP & no & [36] \\
\hline $4 \times 44$ & Homo sapiens & 180 & $\mathrm{AMP}, \mathrm{SO}_{4-;} ; \mathrm{GOL}^{5}$ & R89Q & unpublished \\
\hline $6 \mathrm{FCl}$ & Homo sapiens & 180 & PRP; $\mathrm{ADE} ; \mathrm{Mg}_{2}^{+}$ & no & [37] \\
\hline
\end{tabular}

Table S3. The characteristics of the NT5Es included in the study.

\begin{tabular}{cccccc}
\hline PDB ID & Organism & Residues & $\begin{array}{c}\text { co-crystallized with } \\
\text { substrate or analog } \\
\text { substrate }\end{array}$ & mutations & reference \\
\hline $4 \mathrm{H} 2 \mathrm{I}$ & Homo sapiens & 532 & ${\mathrm{~A} 12^{1} ; \mathrm{Zn}_{2}^{+;} \mathrm{Ca}_{2}^{+;} \mathrm{Cl}^{-}}^{+}$ & no & {$[38]$} \\
$4 \mathrm{H} 2 \mathrm{G}$ & Homo sapiens & 546 & $\mathrm{AND}^{2}, \mathrm{Zn}_{2}^{+;} \mathrm{Ca}_{2}^{+;} \mathrm{Cl}^{-}$ & seven & {$[38]$} \\
$4 \mathrm{H} 2 \mathrm{~F}$ & Homo sapiens & 546 & $\mathrm{AND}^{2}, \mathrm{Zn}_{2}^{+;} \mathrm{Ca}_{2}^{+;} \mathrm{Cl}^{-}$ & seven & {$[38]$} \\
$4 \mathrm{H} 1 \mathrm{~S}$ & Homo sapiens & 530 & $\mathrm{NAG}^{3} ; \mathrm{PO}_{4}^{-} ; \mathrm{Zn}_{2}^{+}$ & no & {$[39]$} \\
\hline
\end{tabular}

${ }^{1}$ phosphomethylphosphonic acid adenosyl ester; ${ }^{2}$ adenosine; ${ }^{3} \mathrm{~N}$-actetyl-D-glucosamine 
Table S4. The characteristics of the NDK3s included in the study.

\begin{tabular}{cccccc}
\hline PDB ID & Organism & Residues & $\begin{array}{c}\text { co-crystallized with } \\
\text { substrate or analog } \\
\text { substrate }\end{array}$ & mutations & reference \\
\hline 1ZS6 & Homo sapiens & 169 & $\mathrm{ADP}$ & no & unpublished \\
2HVD & Homo sapiens & 152 & $\mathrm{ADP}$ & no & {$[40]$} \\
2HVE & Homo sapiens & 152 & $\mathrm{ADP}$ & $\mathrm{S} 120 \mathrm{~B}$ & {$[40]$} \\
$1 \mathrm{JXV}$ & Homo sapiens & 152 & $\mathrm{no}$ & no & {$[41]$} \\
3BBB & Homo sapiens & 151 & $\mathrm{DG}^{2} ; \mathrm{DA}^{3}$ & no & {$[42]$} \\
\hline
\end{tabular}

${ }^{1}$ adenosine- $5^{\prime}$-diphosphate; ${ }^{2} 2^{\prime}$-deoxyguanosine- $5^{\prime}$-monophosphate; ${ }^{3} 2^{\prime}$-deoxyadenosine $-5^{\prime}$-monophosphate

Table S5. The characteristics of the PNP1s included in the study.

\begin{tabular}{cccccc}
\hline PDB ID & Organism & Residues & $\begin{array}{c}\text { co-crystallized with } \\
\text { substrate or analog } \\
\text { substrate }\end{array}$ & mutations & reference \\
\hline 1ULA & Homo sapiens & 289 & $\mathrm{SO}_{4}^{-}$ & no & {$[43]$} \\
1ULB & Homo sapiens & 289 & guanine; $\mathrm{SO}_{4}^{-}$ & no & {$[43]$} \\
2AOW & Homo sapiens & 289 & $\mathrm{DIH}^{1} ; \mathrm{SO}_{4}^{-}$ & $\mathrm{H} 257 \mathrm{G}$ & {$[44]$} \\
2AOX & Homo sapiens & 289 & $\mathrm{DIH}^{1} ; \mathrm{SO}_{4}^{-}$ & $\mathrm{H} 257 \mathrm{~F}$ & {$[44]$} \\
2AOY & Homo sapiens & 289 & $\mathrm{DIH}^{1} ; \mathrm{SO}_{4}^{-}$ & $\mathrm{H} 257 \mathrm{D}$ & {$[44]$} \\
1RSZ & Homo sapiens & 289 & $\mathrm{DIH}^{1} ; \mathrm{SO}_{4}^{-}$ & no & unpublished \\
1RFG & Homo sapiens & 288 & guanosine; $\mathrm{SO}_{4}^{-}$ & no & [45] \\
\hline${ }^{1}$ 7-[[(3R,4R)-3-(hydroxymethyl)-4-oxidanyl-pyrrolidin-1-ium-1-yl]methyl]-3,5-dihydropyrrolo[3,2-d]pyrimidin-4-one
\end{tabular}

Table S6. The characteristics of the CKs included in the study.

\begin{tabular}{|c|c|c|c|c|c|}
\hline PDB ID & Residues & Organism & $\begin{array}{c}\text { co-crystallized with } \\
\text { substrate or analog } \\
\text { substrate }\end{array}$ & mutations & reference \\
\hline \multicolumn{6}{|c|}{ mitochondrial CK } \\
\hline 1CRK & 380 & Gallus gallus & $\mathrm{SO}_{4}^{-}$ & no & {$[46]$} \\
\hline $4 Z 9 M$ & 392 & Homo sapiens & $\mathrm{ADP}^{1}$ & no & unpublished \\
\hline \multicolumn{6}{|c|}{ muscle CK } \\
\hline 2CRK & 381 & $\begin{array}{l}\text { Oryctolagus } \\
\text { cuniculus }\end{array}$ & $\mathrm{SO}_{4}^{-}$ & no & [48] \\
\hline $1 \mathrm{IOE}$ & 381 & Homo sapiens & no & no & [48] \\
\hline $1 U 6 R$ & 380 & $\begin{array}{c}\text { Oryctolagus } \\
\text { cuniculus }\end{array}$ & $\mathrm{ADP} ; \mathrm{IOM}^{2} ; \mathrm{NO}_{3}^{-} ; \mathrm{Mg}_{2+}$ & R134K & [49] \\
\hline \multicolumn{6}{|l|}{ brain CK } \\
\hline 3DRB & 381 & Homo sapiens & $\mathrm{ADP} ; \mathrm{Mg}_{2+}$ & no & {$[50]$} \\
\hline 3DRE & 381 & Homo sapiens & no & no & {$[50]$} \\
\hline 1QH4 & 380 & Gallus gallus & acetate ion; $\mathrm{Ca}_{2}^{+}$ & no & [51] \\
\hline \multicolumn{6}{|c|}{ retinal CK } \\
\hline 1G0W & 380 & Bos taurus & $\mathrm{SO}_{4}^{-}$ & no & [52] \\
\hline
\end{tabular}

